\title{
Goldstino spectrum in an ultracold Bose-Fermi mixture with explicitly broken supersymmetry
}

\author{
Hiroyuki Tajima $\odot,{ }^{1,2, *}$ Yoshimasa Hidaka $\odot,{ }^{2,3}$ and Daisuke Satow ${ }^{4}$ \\ ${ }^{1}$ Department of Mathematics and Physics, Kochi University, Kochi 780-8520, Japan \\ ${ }^{2}$ RIKEN Nishina Center, Wako, Saitama 351-0198, Japan \\ ${ }^{3}$ RIKEN iTHEMS, Wako, Saitama 351-0198, Japan \\ ${ }^{4}$ Arithmer Inc., Minato-Ku, Tokyo 106-6040, Japan
}

(Received 23 January 2020; accepted 3 December 2020; published 12 January 2021)

\begin{abstract}
We theoretically investigate a supersymmetric collective mode called the Goldstino in a Bose-Fermi mixture. The explicit supersymmetry breaking, which is unavoidable in cold-atom experiments, is considered. We derive the Gell-Mann-Oakes-Renner (GOR) relation for the Goldstino, which gives the relation between the energy gap at zero momentum and the explicit breaking term. We also numerically evaluate the gap of the Goldstino above the Bose-Einstein condensation temperature within the random phase approximation (RPA). While the gap obtained from the GOR relation coincides with that in the RPA for the mass-balanced system, there is a deviation from the GOR relation in the mass-imbalanced system. We point out that the deviation becomes large when the Goldstino pole is close to the branch point, although it is parametrically a higher order with respect to the mass-imbalanced parameter. To examine the existence of the Goldstino pole in realistic cold atomic systems, we show how the mass-imbalance effect appears in ${ }^{6} \mathrm{Li}-{ }^{7} \mathrm{Li},{ }^{40} \mathrm{~K}-{ }^{41} \mathrm{~K}$, and ${ }^{173} \mathrm{Yb}-{ }^{174} \mathrm{Yb}$ mixtures. Furthermore, we analyze the Goldstino spectral weight in ${ }^{173} \mathrm{Yb}-{ }^{174} \mathrm{Yb}$ mixture with realistic interactions and show a clear peak due to the Goldstino pole. As a possibility to observe the Goldstino spectrum in cold-atom experiments, we discuss the effects of the Goldstino pole on fermionic single-particle excitation as well as the relationship between the GOR relation and Tan's contact.
\end{abstract}

DOI: 10.1103/PhysRevResearch.3.013035

\section{INTRODUCTION}

Supersymmetry is symmetry with respect to an interchange between bosons and fermions [1-3]. While the existence of supersymmetry is expected in the context of particle physics, evidence or any indications of it have not yet been observed in high-energy experiments [4]. However, apart from whether or not supersymmetric partners such as squarks exist in our world, it is a really interesting problem to explore the consequences of supersymmetry using fermions and bosons, which are well established in condensed matter physics.

An ultracold atomic gas is currently one of the most useful systems for investigating quantum many-body phenomena, due to the controllability of physical parameters such as interaction, density, temperature, and quantum statistical properties of atoms by use of isotopes [5-7]. In particular, the Feshbach resonance [8] enables us to investigate this atomic system from the weak-coupling to the strong-coupling limit in a systematic manner. In this regard, the supersymmetric properties of this system have been extensively discussed theoretically [9-16].

\footnotetext{
*hiroyuki.tajima@riken.jp

Published by the American Physical Society under the terms of the Creative Commons Attribution 4.0 International license. Further distribution of this work must maintain attribution to the author(s) and the published article's title, journal citation, and DOI.
}

Recently, Bose-Fermi mixtures with a small mass imbalance between bosons and fermions such as ${ }^{6} \mathrm{Li}-{ }^{7} \mathrm{Li}$ [17-19], ${ }^{39} \mathrm{~K}-{ }^{40} \mathrm{~K}[20],{ }^{40} \mathrm{~K}-{ }^{41} \mathrm{~K}[21],{ }^{84} \mathrm{Sr}-{ }^{87} \mathrm{Sr}[22],{ }^{87} \mathrm{Rb}-{ }^{87} \mathrm{Sr}$ [23], ${ }^{161} \mathrm{Dy}-{ }^{162} \mathrm{Dy}[24]$, and ${ }^{173} \mathrm{Yb}-{ }^{174} \mathrm{Yb}[25,26]$ have been experimentally realized. The boson-boson or boson-fermion interactions in some of the mixtures can be tuned due to the magnetic Feshbach resonance [18,21,23,27-29]. In this sense, examining supersymmetry in such cold atomic systems is promising.

A remarkable feature of supersymmetry in a Bose-Fermi mixture is the emergence of a Nambu-Goldstone (NG) mode called the Goldstino [30-34]. While the usual NG mode propagates as a bosonic mode, the Goldstino behaves as a fermionic mode. Such a fermionic collective excitation has also been predicted in quantum electrodynamics as well as quantum chromodynamics [35-37]. Observation of this collective mode is really important for seeing the supersymmetric properties in a Bose-Fermi mixture that are realized in a tabletop experiment. Since the Goldstino is a fermionic collective mode associated with broken supersymmetry, it becomes a gapless mode when the system possesses exact supersymmetry. However, explicit supersymmetry breaking such as the mass imbalance between fermions and bosons is unavoidable in cold-atom experiments. In this case, the Goldstino has a finite energy gap associated with the explicit breaking parameters. If one can observe the gapped Goldstino and its spectral properties agree with the results of theoretical analysis, this should be evidence for the existence of supersymmetry in these systems. Indeed, the first example of NG bosons in 
particle physics was pions, which are also gapped modes due to the explicitly broken chiral symmetry associated with the current quark mass [38].

In this work, we theoretically examine the energy gap of the Goldstino in a Bose-Fermi mixture with explicitly broken supersymmetry. We focus on a few candidates for nearly supersymmetric Bose-Fermi mixtures, namely, ${ }^{6} \mathrm{Li}-{ }^{7} \mathrm{Li},{ }^{40} \mathrm{~K}-{ }^{41} \mathrm{~K}$, and ${ }^{173} \mathrm{Yb}-{ }^{174} \mathrm{Yb}$ mixtures. We determine the thermodynamic properties of weakly interacting mixtures within the Hartree-Fock mean-field approximation above the Bose-Einstein condensation (BEC) temperature. By developing a gap formula for the Goldstino, which corresponds to the Gell-Mann-Oakes-Renner (GOR) relation in quantum chromodynamics [39], based on the memory function formalism [40], we show how the explicit supersymmetry-breaking terms affect the Goldstino gap in these systems. By comparing it with the numerical results of the random phase approximation (RPA), we clarify that the effects of the branch point are significant in the presence of the mass imbalance between fermions and bosons. Furthermore, we discuss how to observe the Goldstino gap from the single-particle spectral function of a Fermi atom. While the previous work done by two of the authors is dedicated to the two-dimensional system [14] and the three-dimensional one in the BEC phase [15] with ideal situations such as supersymmetric interactions, in this paper we discuss the three-dimensional system with realistic physical parameters above the Bose-Einstein condensation temperature $T_{\mathrm{BEC}}$. Furthermore, we consider the case with a mass imbalance where the fermionic mass is slightly lighter than the bosonic one. In this case, the effects of the branch point are more important. We show that, even when the mass imbalance is small, the peak of the Goldstino disappears and it is buried in the continuum spectrum if the interaction is too weak. We also note that there is a work which considered a mass-imbalanced Bose-Fermi system [13] in which a BoseFermi mixture trapped on an optical lattice was considered, and the species of atoms were not specified. These points are improved in this paper.

This paper is organized as follows: In Sec. II, we introduce our model and the formulation for thermodynamic quantities and the Goldstino gap within the GOR and RPA. In Sec. III, we show and discuss our numerical results within the RPA on the Goldstino gap in a few Bose-Fermi mixture systems. Section IV is devoted to discussion of how the Goldstino pole can affect the fermionic single-particle spectrum, in order to suggest the possibility of detecting the Goldstino in experiments. We summarize our studies in Sec. V. In Appendix A, we show the detailed derivation of the GOR relation based on the memory function formalism. We calculate the Goldstino spectral function in the free limit in Appendix B to check the absence of numerical artifacts. In Appendix C, we discuss the cutoff dependence of the fermionic single-particle spectral function.

\section{FORMALISM}

\section{A. Model}

We consider a nonrelativistic Bose-Fermi mixture described by the Hamiltonian

$$
\begin{aligned}
H= & \int d^{3} \boldsymbol{r} \psi_{b}^{\dagger}(\boldsymbol{r})\left(-\frac{\nabla^{2}}{2 m_{b}}-\mu_{b}\right) \psi_{b}(\boldsymbol{r}) \\
& +\int d^{3} \boldsymbol{r} \psi_{f}^{\dagger}(\boldsymbol{r})\left(-\frac{\nabla^{2}}{2 m_{f}}-\mu_{f}\right) \psi_{f}(\boldsymbol{r}) \\
& +\frac{U_{b b}}{2} \int d^{3} \boldsymbol{r} \psi_{b}^{\dagger}(\boldsymbol{r}) \psi_{b}^{\dagger}(\boldsymbol{r}) \psi_{b}(\boldsymbol{r}) \psi_{b}(\boldsymbol{r}) \\
& +U_{b f} \int d^{3} \boldsymbol{r} \psi_{b}^{\dagger}(\boldsymbol{r}) \psi_{b}(\boldsymbol{r}) \psi_{f}^{\dagger}(\boldsymbol{r}) \psi_{f}(\boldsymbol{r}),
\end{aligned}
$$

where $\psi_{b(f)}$ is the field operator of a boson (fermion) with mass $m_{b(f)}$ and chemical potential $\mu_{b(f)}$. $U_{b b(b f)}$ is the coupling constant of a boson-boson (boson-fermion) interaction, which is assumed to be a contact type. These coupling constants are related to the scattering length $a_{b b(b f)}$ as $U_{b b}=$ $\left(4 \pi a_{b b}\right) / m_{b}$ and $U_{b f}=\left(2 \pi a_{b f}\right) / m_{r}$, respectively, where $m_{r}=$ $1 /\left(1 / m_{f}+1 / m_{b}\right)$ is the reduced mass. In this paper, we measure the interaction strength by using the dimensionless parameters $k_{b} a_{b b}$ and $k_{b} a_{b f}$, where $k_{b}=\left(6 \pi^{2} N_{b}\right)^{1 / 3}$ is a momentum scale for the boson density $N_{b}$. In general, there is a non- $s$-wave fermion-fermion interaction such as the dipoledipole interaction given by

$$
V_{f f}=\frac{1}{2} \int d^{3} \boldsymbol{r} \psi_{f}^{\dagger}(\boldsymbol{r}) \psi_{f}^{\dagger}\left(\boldsymbol{r}^{\prime}\right) U_{f f}\left(\boldsymbol{r}-\boldsymbol{r}^{\prime}\right) \psi_{f}\left(\boldsymbol{r}^{\prime}\right) \psi_{f}(\boldsymbol{r}) .
$$

Although it is negligible in several Fermi atoms such as ${ }^{6} \mathrm{Li}$ and ${ }^{40} \mathrm{~K}$ far away from higher partial-wave Feshbach resonances like a $p$-wave resonance [41] at low temperature, the scattering in these higher partial waves would be important at high enough temperatures [42]. Moreover, the dipole-dipole interaction would become significant in a ${ }^{161} \mathrm{Dy}$ - ${ }^{162} \mathrm{Dy}$ mixture with large magnetic dipole moments [24]. In this work, we consider the case in which these interactions are negligible for simplicity. We note that the intercomponent interaction $U_{b f}$ involves a factor 2 , in contrast to the intracomponent interaction $U_{b b}$ [43]. When $m_{f}=m_{b}, \mu_{b}=\mu_{f}$, and $U_{b b}=$ $U_{b f}$, there is a supersymmetry corresponding to interchange between bosons and fermions: $\psi_{b} \rightarrow \psi_{f}$ and $\psi_{f} \rightarrow \psi_{b}$. The corresponding Noether charges are

$$
Q=\int d^{3} \boldsymbol{r} q(\boldsymbol{r}), \quad Q^{\dagger}=\int d^{3} \boldsymbol{r} q^{\dagger}(\boldsymbol{r}),
$$

which commute with the Hamiltonian, $[H, Q]=\left[H, Q^{\dagger}\right]=0$. Here, $q(\boldsymbol{r})=\psi_{f}(\boldsymbol{r}) \psi_{b}^{\dagger}(\boldsymbol{r})$ is the local operator that creates the boson and annihilates the fermion [10]. Unlike the supersymmetry in relativistic systems, the anticommutation relation between supercharges is not the Hamiltonian but the total particle number operator:

$$
\left\{Q, Q^{\dagger}\right\}=\int d^{3} \boldsymbol{r} \psi_{f}^{\dagger}(\boldsymbol{r}) \psi_{f}(\boldsymbol{r})+\int d^{3} \boldsymbol{r} \psi_{b}^{\dagger}(\boldsymbol{r}) \psi_{b}(\boldsymbol{r}) .
$$

In this sense, the supersymmetry in a nonrelativistic BoseFermi mixture is a different type from that in relativistic theories. The order parameter of supersymmetry breaking is the total number density, $\left\langle\left\{Q, q^{\dagger}(\boldsymbol{r})\right\}\right\rangle=\left\langle\psi_{b}^{\dagger}(\boldsymbol{r}) \psi_{b}(\boldsymbol{r})\right\rangle+$ $\left\langle\psi_{f}^{\dagger}(\boldsymbol{r}) \psi_{f}(\boldsymbol{r})\right\rangle$, which is always broken in a finite-density system. For a spontaneous breaking of bosonic continuous symmetry, if the order parameter is expressed as the expectation value of the commutation relation between a charge 
and a charge density, the breaking pattern is called type B [44-49]. On the other hand, if no such order parameter exists, the breaking pattern is called type A. The NG modes corresponding to type $\mathrm{B}$ typically exhibit a quadratic dispersion. A typical example of a type B NG mode is the magnon in a ferromagnet, in which the order parameter is expressed as the expectation value of the commutation relation between spins. Replacing the commutator with the anticommutator, we can identify the supersymmetry breaking pattern as type B. As in an ordinary symmetry breaking, the supersymmetry breaking leads to a gapless excitation. If the excitation can be identified as a single-mode excitation, it is called the Goldstino. In general, the excitation may be located at a branch point where a two- or multiparticle continuum starts. This is especially the case for the noninteracting system, where there is no Goldstino. The excitation is the particle-hole one. The interaction plays an important role in the existence of the Goldstino. In the following analysis, we assume the existence of Goldstino excitation, and we numerically check it in the RPA in Sec. III. Since the order parameter is expressed as the expectation value of the anticommutation relation of the supercharge and its density, the Goldstino belongs to the type B mode [44-49], which typically has a quadratic dispersion.

In a realistic situation, the supersymmetry is explicitly broken because all parameters cannot be exactly tuned in experiments. The effect of the explicit breaking can be expressed as the commutation relation between the Hamiltonian and the supercharge,

$$
\begin{aligned}
{[H, Q]=} & \int d^{3} \boldsymbol{r} \psi_{b}^{\dagger}(\boldsymbol{r})\left(\chi \frac{\nabla^{2}}{2 m_{r}}+\Delta \mu\right) \psi_{f}(\boldsymbol{r}) \\
& -\Delta U \int d^{3} \boldsymbol{r} \psi_{b}^{\dagger}(\boldsymbol{r}) \psi_{b}^{\dagger}(\boldsymbol{r}) \psi_{b}(\boldsymbol{r}) \psi_{f}(\boldsymbol{r}),
\end{aligned}
$$

where we define

$$
\begin{aligned}
\Delta \mu & \equiv \mu_{f}-\mu_{b}, \\
\chi & \equiv\left(\frac{1}{m_{f}}-\frac{1}{m_{b}}\right) m_{r}=\frac{m_{b}-m_{f}}{m_{b}+m_{f}}, \\
\Delta U & \equiv U_{b f}-U_{b b} .
\end{aligned}
$$

These explicit breakings cause a finite gap of the Goldstino, whose formula is shown in the next subsection.

\section{B. Gell-Mann-Oakes-Renner relation}

Pions are the NG bosons associated with the spontaneous breaking of chiral symmetry in quantum chromodynamics. The GOR formula relates the pion mass and the current quark mass that explicitly breaks chiral symmetry [39]. We can generalize the GOR relation to that of Goldstino in a Bose-Fermi mixture. For this purpose, we employ the memory function formalism [40], which is a different formalism from the one used in the original derivation [39]. The derivation is slightly technical, so that here we show the only result. For readers who are interested in the derivation, see Appendix A.

We consider the retarded Goldstino propagator, defined as

$$
\Gamma^{R}(\boldsymbol{r}, t) \equiv i \theta(t)\left\langle\left\{q(\boldsymbol{r}, t), q^{\dagger}(\mathbf{0}, 0)\right\}\right\rangle .
$$

After Fourier transformation, we obtain

$$
\Gamma^{R}(\boldsymbol{p}, \omega)=i \int_{-\infty}^{\infty} d t \int d^{3} \boldsymbol{r} e^{i \omega t-i \boldsymbol{p} \cdot \boldsymbol{r}} \theta(t)\left\langle\left\{q(\boldsymbol{r}, t), q^{\dagger}(\mathbf{0}, 0)\right\}\right\rangle .
$$

The energy gap is obtained from the pole of $\Gamma^{R}(\boldsymbol{p}, \omega)$ in the complex $\omega$ plane. Since we are interested in the zero-momentum gap of Goldstino, hereafter we take $\boldsymbol{p}=\mathbf{0}$. The memory function formalism systematically decomposes $\Gamma^{R}(\omega)$ into the form

$$
\Gamma^{R}(\omega)=\frac{-N}{\omega+\Omega+i \Phi(\omega)},
$$

where $N=\left\langle\psi_{f}^{\dagger}(\boldsymbol{r}) \psi_{f}(\boldsymbol{r})\right\rangle+\left\langle\psi_{b}^{\dagger}(\boldsymbol{r}) \psi_{b}(\boldsymbol{r})\right\rangle$ is the total number density. $\Phi(\omega)$ and $\Omega=\left\langle\left\{[H, Q], q^{\dagger}(\mathbf{0}, 0)\right\}\right\rangle / N$ are called the dynamic and static parts of the memory function. We do not show the explicit form of $\Phi(\omega)$; the important point is that $\Phi(\omega)$ is parametrically higher oder compared with $\Omega$ with respect to the explicit breaking term (see Appendix A for more details). Therefore, at the leading order of the explicit breaking term, the energy gap $\omega_{G}$ is expressed as

$$
\omega_{G}=\omega_{G}^{\mathrm{GOR}} \equiv-\frac{1}{N}\left\langle\left\{[H, Q], q^{\dagger}(\mathbf{0}, 0)\right\}\right\rangle .
$$

We emphasize that this formula works for any supersymmetric Hamiltonian with a small explicit breaking term and local interactions because we have not employed the specific form of the Hamiltonian. The gap is linearly proportional to the explicit breaking term, whose property can be understood as type B breaking [44-49]. In contrast, type A breaking predicts that the gap is proportional to the square root of the explicit breaking term. We note that although the dynamic part $\Phi(\omega)$ is higher order, it might not be small if there is a singularity in $\Phi(\omega)$. As shown later, this is the case when the branch point is close to $\omega_{G}^{\mathrm{GOR}}$.

For the Hamiltonian, (1), that we employ in the present paper, using Eq. (5), we obtain

$$
\begin{aligned}
\left\{[H, Q], q^{\dagger}(\boldsymbol{r}, 0)\right\}= & \frac{\chi}{2 m_{r}}\left[\left\{\nabla^{2} \psi_{b}^{\dagger}(\boldsymbol{r})\right\} \psi_{b}(\boldsymbol{r})+\psi_{f}^{\dagger}(\boldsymbol{r}) \nabla^{2} \psi_{f}(\boldsymbol{r})\right] \\
& +\Delta \mu\left[\psi_{b}^{\dagger}(\boldsymbol{r}) \psi_{b}(\boldsymbol{r})+\psi_{f}^{\dagger}(\boldsymbol{r}) \psi_{f}(\boldsymbol{r})\right] \\
& -\Delta U\left[\psi_{b}^{\dagger}(\boldsymbol{r}) \psi_{b}^{\dagger}(\boldsymbol{r}) \psi_{b}(\boldsymbol{r}) \psi_{b}(\boldsymbol{r})\right. \\
& \left.+2 \psi_{b}^{\dagger}(\boldsymbol{r}) \psi_{b}(\boldsymbol{r}) \psi_{f}^{\dagger}(\boldsymbol{r}) \psi_{f}(\boldsymbol{r})\right] .
\end{aligned}
$$

Therefore, the Goldstino gap in the present model is given by

$$
\begin{aligned}
\omega_{G}^{\mathrm{GOR}}= & -\Delta \mu-\frac{\chi}{2 m_{r} N}\left[\left\langle\left\{\nabla^{2} \psi_{b}^{\dagger}(\boldsymbol{r})\right\} \psi_{b}(\boldsymbol{r})+\psi_{f}^{\dagger}(\boldsymbol{r}) \nabla^{2} \psi_{f}(\boldsymbol{r})\right\rangle\right] \\
& +\frac{\Delta U}{N}\left[\left\langle\psi_{b}^{\dagger}(\boldsymbol{r}) \psi_{b}^{\dagger}(\boldsymbol{r}) \psi_{b}(\boldsymbol{r}) \psi_{b}(\boldsymbol{r})\right\rangle\right. \\
& \left.+2\left\langle\psi_{b}^{\dagger}(\boldsymbol{r}) \psi_{b}(\boldsymbol{r}) \psi_{f}^{\dagger}(\boldsymbol{r}) \psi_{f}(\boldsymbol{r})\right\rangle\right] \\
= & -\Delta \mu+\chi\langle\mathcal{E}\rangle+\frac{2 \Delta U}{U}\langle V\rangle,
\end{aligned}
$$

where $U \equiv\left(U_{b f}+U_{b b}\right) / 2$. Here $\langle\mathcal{E}\rangle$ and $\langle V\rangle$ are the average kinetic and interaction energy of one particle per volume:

$$
\langle\mathcal{E}\rangle=\frac{1}{N}\left\langle\psi_{b}^{\dagger}(\boldsymbol{r}) \frac{-\nabla^{2}}{2 m_{r}} \psi_{b}(\boldsymbol{r})+\psi_{f}^{\dagger}(\boldsymbol{r}) \frac{-\nabla^{2}}{2 m_{r}} \psi_{f}(\boldsymbol{r})\right\rangle,
$$




$$
\begin{aligned}
\langle V\rangle= & \frac{1}{N}\left\langle\frac{U}{2} \psi_{b}^{\dagger}(\boldsymbol{r}) \psi_{b}^{\dagger}(\boldsymbol{r}) \psi_{b}(\boldsymbol{r}) \psi_{b}(\boldsymbol{r})\right. \\
& \left.+U \psi_{b}^{\dagger}(\boldsymbol{r}) \psi_{b}(\boldsymbol{r}) \psi_{f}^{\dagger}(\boldsymbol{r}) \psi_{f}(\boldsymbol{r})\right\rangle .
\end{aligned}
$$

Here, we take the limits of $\chi \rightarrow 0\left(m_{b} \rightarrow m_{f}\right)$ and $\Delta U \rightarrow 0$ $\left(U_{b b} \rightarrow U_{b f}\right)$ to suppress the higher-order breaking terms being proportional to $\chi^{2}$ and $\Delta U^{2}$ when we define $\langle\mathcal{E}\rangle$ and $\langle V\rangle$ in Eqs. (15) and (16). These parameters can also be expressed by the pressure $P\left(T, \mu, m_{f}, m_{b}, U_{b b}, U_{b f}\right)$ as a function of $T$, $\mu, m_{f}, m_{b}, U_{b b}$, and $U_{b f}$ :

$$
\begin{aligned}
\langle\mathcal{E}\rangle & =\frac{1}{m_{r}}\left(m_{f}^{2} \frac{\partial P}{\partial m_{f}}+m_{b}^{2} \frac{\partial P}{\partial m_{b}}\right), \\
\langle V\rangle & =-U\left(\frac{\partial P}{\partial U_{b f}}+\frac{\partial P}{\partial U_{b b}}\right) .
\end{aligned}
$$

We note that this result is correct up to first order in explicit symmetry breaking, and we did not use any approximations such as the RPA in its derivation. We also note that the expectation values of the local operator $\left\langle\psi_{b}^{\dagger}(\boldsymbol{r}) \psi_{b(f)}^{\dagger}(\boldsymbol{r}) \psi_{b}(\boldsymbol{r}) \psi_{b(f)}(\boldsymbol{r})\right\rangle$ in zero-range models are known to be associated with the so-called Tan's contact $C_{b b(b f)}[50-53]$ as

$$
\langle V\rangle=\frac{U}{N}\left[\frac{C_{b f}}{\left(4 \pi a_{b f}\right)^{2}}+\frac{C_{b b}}{\left(4 \pi a_{b b}\right)^{2}}\right] .
$$

The universal relations with respect to this quantity are expected to hold even in the weakly repulsive case $[54,55]$. Indeed, $C_{b b}$ is analytically obtained within the mean-field Bogoliubov theory at $T=0$ in Refs. [54] and [56]. The GOR relation is therefore rewritten as

$$
\begin{aligned}
\omega_{G}^{\mathrm{GOR}} & =-\Delta \mu+\chi\langle\mathcal{E}\rangle+2 \frac{\Delta U}{N}\left[\frac{C_{b f}}{\left(4 \pi a_{b f}\right)^{2}}+\frac{C_{b b}}{\left(4 \pi a_{b b}\right)^{2}}\right] \\
& =-\Delta \mu+\chi\langle\mathcal{E}\rangle+\frac{1}{2 \pi N}\left(\frac{a_{b f}}{2 m_{r}}-\frac{a_{b b}}{m_{b}}\right)\left(\frac{C_{b f}}{a_{b f}^{2}}+\frac{C_{b b}}{a_{b b}^{2}}\right) .
\end{aligned}
$$

Since Tan's contact can be observed precisely, this relation is also useful to address the Goldstino properties in recent experiments. However, a strong repulsive interaction beyond the present weak-coupling mean-field approximation generally involves an effective range correction acting as a momentum cutoff to avoid an ultraviolet divergence in a three-dimensional system $[57,58]$. In this case, one has to extend Eq. (20) to a relation with the effective ranges of the interactions. It is particularly important to develop the relation between the GOR relation and the high-momentum tails of the distribution functions [51], which is left for future work. In this paper, we restrict ourselves to the weak-coupling regime. Since we assume a homogeneous case with translational symmetry, we can take $r \rightarrow 0$ taking $\nabla^{2}$ in the terms in $\langle\mathcal{E}\rangle$. We also note that the GOR relation derived in this paper is valid both below and above the Bose-Einstein condensation temperature $T_{\mathrm{BEC}}$. To address the BEC phase below $T_{\mathrm{BEC}}$, one has to take the mean-field term associated with the condensate into account [59]. In this paper, we consider the normal phase above $T_{\mathrm{BEC}}$ for simplicity.

\section{Mean-field approximation}

In this paper, we employ the weak-coupling mean-field approximation to calculate the Goldstino gap by using the GOR relation, (14). At a weak coupling, the thermal average with respect to the interaction term in Eq. (14) can be approximated as

$$
\begin{aligned}
&\left\langle\psi_{b}^{\dagger}(\boldsymbol{r}) \psi_{b}^{\dagger}(\boldsymbol{r}) \psi_{b}(\boldsymbol{r}) \psi_{b}(\boldsymbol{r})\right\rangle \simeq 2 N_{b}^{2}, \\
&\left\langle\psi_{b}^{\dagger}(\boldsymbol{r}) \psi_{b}(\boldsymbol{r}) \psi_{f}^{\dagger}(\boldsymbol{r}) \psi_{f}(\boldsymbol{r})\right\rangle \simeq N_{b} N_{f},
\end{aligned}
$$

where the particle number densities $N_{b(f)}$ are obtained as

$$
\begin{aligned}
& N_{b}=\int \frac{d^{3} \boldsymbol{q}}{(2 \pi)^{3}} n_{b}\left(\xi_{\boldsymbol{q}}^{b}\right), \\
& N_{f}=\int \frac{d^{3} \boldsymbol{k}}{(2 \pi)^{3}} n_{f}\left(\xi_{\boldsymbol{k}}^{f}\right),
\end{aligned}
$$

where $n_{b(f)}(x)=1 /(\exp (x / T) \mp 1)$ is the Bose (Fermi) distribution function. Here we have defined $\xi_{q}^{b}=q^{2} /\left(2 m_{b}\right)-\mu_{b}+$ $\Sigma_{b}^{H}$ and $\xi_{\boldsymbol{k}}^{f}=k^{2} /\left(2 m_{f}\right)-\mu_{f}+\Sigma_{f}^{H}$. The Hartree shift $\Sigma_{b(f)}^{H}$ is given by

$$
\begin{gathered}
\Sigma_{b}^{H}=2 U_{b b} N_{b}+U_{b f} N_{f}, \\
\Sigma_{f}^{H}=U_{b f} N_{b} .
\end{gathered}
$$

Substituting Eqs. (23) and (24) into Eq. (14), one obtains

$$
\omega_{G}^{\mathrm{GOR}}=-\Delta \mu+\chi\langle\mathcal{E}\rangle_{\mathrm{HF}}+2 N_{b} \Delta U .
$$

Here, we have defined

$$
\langle\mathcal{E}\rangle_{\mathrm{HF}} \equiv \frac{1}{N} \int \frac{d^{3} \boldsymbol{k}}{(2 \pi)^{3}}\left[n_{b}\left(\xi_{\boldsymbol{k}}^{b}\right)+n_{f}\left(\xi_{\boldsymbol{k}}^{f}\right)\right] \frac{k^{2}}{2 m_{r}} .
$$

In particular, in the mass-balanced case $\left(m_{b}=m_{f}\right)$ relevant for a ${ }^{87} \mathrm{Sr}-{ }^{87} \mathrm{Rb}$ mixture, one finds

$$
\omega_{G}^{\mathrm{GOR}}=-\Delta \mu+2 N_{b} \Delta U .
$$

Our result agrees with the result in Ref. [12] obtained in a tight-binding model. We note that Eq. (29) obtained in the normal phase is different from the result in Ref. [16], which considered the BEC phase at zero temperature. We also note that in the mean-field approximation one can obtain Tan's contacts as $C_{b f}=16 \pi^{2} a_{b f}^{2} N_{b} N_{f}$ and $C_{b b}=16 \pi^{2} a_{b b}^{2} N_{b}^{2}$. One can reproduce Eq. (27) by substituting them into Eq. (20).

\section{Random phase approximation}

We compare the results of the GOR relation with the RPA calculation to see the effects of continuum and higher-order correction in the explicit breaking term. In Ref. [13], it is reported that the imbalance of the hopping amplitude in the lattice model (which corresponds to the mass imbalance in the present case) generates the continuum mode in addition to the Goldstino pole, within the RPA. In this work, we address how the GOR relation works in the presence of such a continuum. We consider the series of fermion-boson bubble $\Pi$ diagrammatically described in Fig. 1. The explicit form of the Goldstino propagator $\Gamma^{R}$ reads

$$
\Gamma^{R}(\boldsymbol{p}, \omega)=\frac{\Pi(\boldsymbol{p}, \omega)}{1+U_{b f} \Pi(\boldsymbol{p}, \omega)},
$$




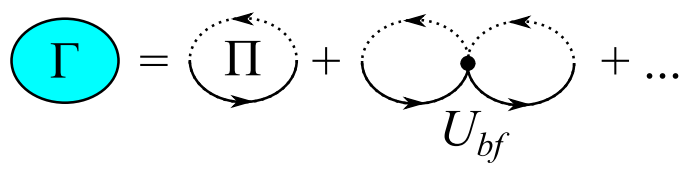

FIG. 1. Feynman diagrams for the Goldstino propagator $\Gamma$ consisting of RPA series of boson-fermion bubble П. The solid (dashed) line and the black dot represent the fermion (boson) propagator and the boson-fermion interaction $U_{b f}$, respectively.

where

$$
\Pi(\boldsymbol{p}, \omega)=-\int \frac{d^{3} \boldsymbol{k}}{(2 \pi)^{3}} \frac{n_{f}\left(\xi_{\boldsymbol{k}}^{f}\right)+n_{b}\left(\xi_{\boldsymbol{k}-\boldsymbol{p}}^{b}\right)}{\omega+i \delta+\xi_{\boldsymbol{k}-\boldsymbol{p}}^{b}-\xi_{\boldsymbol{k}}^{f}}
$$

is a bubble diagram with respect to the fermion-boson exchange. Here $\delta$ is an infinitesimally small value. In the numerical calculations, $\delta$ is taken to be $10^{-3} \varepsilon_{b}$ in this paper. Here $\varepsilon_{b}=\left(6 \pi^{2} N_{b}\right)^{2 / 3} /\left(2 m_{b}\right)$ is the energy scale associated with the boson density $N_{b}$. We note that the continuum is generated when the kinematics of 1 to 2 scattering is possible for multiple $\omega$ due to multiple $\boldsymbol{k}$ :

$$
\omega+\xi_{k-p}^{b}=\xi_{k}^{f} .
$$

For $\boldsymbol{p}=\mathbf{0}$ and $\chi>0$, the branch point is located at $\omega_{\mathrm{BP}}=\xi_{\mathbf{0}}^{f}-\xi_{\mathbf{0}}^{b}=-\Delta \mu+2 \Delta U N_{b}-U_{b f} N$. There is a continuum spectrum for $\omega \geqslant \omega_{\mathrm{BP}}$. Since $\omega_{\mathrm{BP}}$ can be written as $\omega_{\mathrm{BP}}=\omega_{G}^{\mathrm{GOR}}-\chi\langle\mathcal{E}\rangle_{\mathrm{HF}}-U_{b f} N, \omega=\omega_{G}^{\mathrm{GOR}}$ is always in the continuum for $\chi>0$. In contrast, when $\chi<0$, the continuum spectrum exists for $\omega \leqslant \omega_{\mathrm{BP}}$. Thus, $\omega=\omega_{G}^{\mathrm{GOR}}$ is in the continuum when $U_{b f} N \leqslant-\chi\langle\mathcal{E}\rangle_{\mathrm{HF}}$.

The Goldstino gap is obtained by the zero point of the denominator of $\Gamma^{R}(\mathbf{0}, \omega)$, i.e., $1+U_{b f} \Pi(\mathbf{0}, \omega)=0$. In the mass-balanced case, one can analytically estimate the Goldstino gap from

$$
\begin{aligned}
0 & =1+U_{b f} \Pi\left(\mathbf{0}, \omega_{G}\right) \\
& =1-U_{b f} \int \frac{d^{3} \boldsymbol{k}}{(2 \pi)^{3}} \frac{n_{f}\left(\xi_{\boldsymbol{k}}^{f}\right)+n_{b}\left(\xi_{\boldsymbol{k}}^{b}\right)}{\omega_{G}+i \delta+\Delta \mu-2 \Delta U N_{b}+U_{b f} N} \\
& =\frac{\omega_{G}+\Delta \mu-2 \Delta U N_{b}}{\omega_{G}+i \delta+\Delta \mu-2 \Delta U N_{b}+U_{b f} N},
\end{aligned}
$$

and therefore

$$
\omega_{G}=-\Delta \mu+2 N_{b} \Delta U .
$$

We note that the $\boldsymbol{k}$ dependence completely vanishes from Eq. (32) at $\boldsymbol{p}=\mathbf{0}$, and therefore the width of the continuum becomes 0. Equation (34) coincides with the GOR relation given by Eq. (29) [13-15]. Beyond the RPA, there will be corrections coming from interactions between quasiparticles. Since the differences in chemical potentials and interactions simply induces a shift of the Goldstino pole, the supersymmetric collective mode can be confirmed experimentally by checking the interaction and chemical potential dependences of the gap in a weakly interacting mass-balanced mixture.

On the other hand, in the presence of the mass imbalance between bosons and fermions, there is a correction to the GOR relation, which is parametrically higher order in the explicit breaking term. However, the correction may not be small if the branch point is close to $\omega_{G}^{\mathrm{GOR}}$. To see this, we parametrize the denominator of $\Gamma^{R}(\mathbf{0}, \omega)$ as

$$
1+U_{b f} \Pi(\mathbf{0}, \omega)=\frac{1}{U_{b f} N}\left[\omega-\omega_{G}^{\mathrm{GOR}}-\tilde{\Phi}(\omega)\right],
$$

where

$$
\begin{aligned}
\tilde{\Phi}(\omega)= & \frac{1}{N} \int \frac{d^{3} \boldsymbol{k}}{(2 \pi)^{3}}\left[n_{f}\left(\xi_{\boldsymbol{k}}^{f}\right)+n_{b}\left(\xi_{\boldsymbol{k}}^{b}\right)\right] \\
& \times \frac{\left[\omega+\Delta \mu-\chi k^{2} /\left(2 m_{r}\right)-2 \Delta U N_{b}\right]^{2}}{\omega+i \delta-\chi k^{2} /\left(2 m_{r}\right)-\omega_{\mathrm{BP}}} .
\end{aligned}
$$

$\tilde{\Phi}(\omega)$ plays a role similar to that of the dynamic part of the memory function defined in Eq. (A12), although the definition is different. At $\omega=\omega_{G}^{\mathrm{GOR}}, \tilde{\Phi}\left(\omega_{G}^{\mathrm{GOR}}\right)$ is explicitly proportional to $\chi^{2}$ :

$$
\begin{aligned}
\tilde{\Phi}\left(\omega_{G}^{\mathrm{GOR}}\right)= & \chi^{2} \frac{1}{N} \int \frac{d^{3} \boldsymbol{k}}{(2 \pi)^{3}}\left[n_{f}\left(\xi_{\boldsymbol{k}}^{f}\right)+n_{b}\left(\xi_{\boldsymbol{k}}^{b}\right)\right] \\
& \times \frac{\left[k^{2} /\left(2 m_{r}\right)-\langle\mathcal{E}\rangle_{\mathrm{HF}}\right]^{2}}{\omega_{G}^{\mathrm{GOR}}-\omega_{\mathrm{BP}}-\chi k^{2} /\left(2 m_{r}\right)+i \delta} .
\end{aligned}
$$

From this expression, the correction in the $\chi^{2}$ order is evaluated as

$$
\begin{aligned}
\tilde{\Phi}\left(\omega_{G}^{\mathrm{GOR}}\right) \simeq & \frac{\chi^{2}}{U_{b f} N} \frac{1}{N} \int \frac{d^{3} \boldsymbol{k}}{(2 \pi)^{3}}\left[n_{f}\left(\xi_{\boldsymbol{k}}^{f}\right)+n_{b}\left(\xi_{\boldsymbol{k}}^{b}\right)\right] \\
& \times\left(\langle\mathcal{E}\rangle_{\mathrm{HF}}-\frac{k^{2}}{2 m_{r}}\right)^{2} .
\end{aligned}
$$

We can estimate the scale of $\tilde{\Phi}\left(\omega_{G}^{\mathrm{GOR}}\right)$ as $\chi^{2}\langle\mathcal{E}\rangle_{\mathrm{HF}}^{2} /\left(U_{b f} N\right)$, where the integral of $\left(\langle\mathcal{E}\rangle_{\mathrm{HF}}-k^{2} / 2 m_{r}\right)^{2}$ is estimated to be $\langle\mathcal{E}\rangle_{\mathrm{HF}}^{2}$. Since $\chi\langle\mathcal{E}\rangle_{\mathrm{HF}} \sim \omega_{G}^{\mathrm{GOR}}$ and $\omega_{\mathrm{BP}} \sim U_{b f} N$ for a small explicit symmetry-breaking case, we obtain

$$
\tilde{\Phi}\left(\omega_{G}^{\mathrm{GOR}}\right) \sim \omega_{G}^{\mathrm{GOR}}\left|\frac{\omega_{G}^{\mathrm{GOR}}}{\omega_{\mathrm{BP}}}\right| .
$$

Similarly, we can estimate the $n$th order in $\chi$ as of order $\omega_{G}^{\mathrm{GOR}}\left|\omega_{G}^{\mathrm{GOR}} / \omega_{\mathrm{BP}}\right|^{n-1}$. This expansion breaks down if $\left|\omega_{G}^{\mathrm{GOR}} / \omega_{\mathrm{BP}}\right|$ is not small even though $\chi \ll 1$.

When $\chi>0$, there is a contribution from the imaginary part of $\tilde{\Phi}\left(\omega_{G}^{\mathrm{GOR}}\right)$ to the dispersion relation, which can be analytically evaluated as

$$
\begin{aligned}
-\operatorname{Im} \tilde{\Phi}\left(\omega_{G}^{\mathrm{GOR}}\right)= & \chi^{2} \frac{\pi}{N} \int \frac{d^{3} \boldsymbol{k}}{(2 \pi)^{3}}\left[n_{f}\left(\xi_{\boldsymbol{k}}^{f}\right)+n_{b}\left(\xi_{\boldsymbol{k}}^{b}\right)\right] \\
& \times\left(\frac{k^{2}}{2 m_{r}}-\langle\mathcal{E}\rangle_{\mathrm{HF}}\right)^{2} \\
& \times \delta\left(\omega_{G}^{\mathrm{GOR}}-\omega_{\mathrm{BP}}-\chi \frac{k^{2}}{2 m_{r}}\right) \\
= & \frac{U_{b f}}{4 \pi}\left(\frac{2 m_{r} U_{b f} N}{\chi}\right)^{\frac{3}{2}} \sqrt{1+\chi \frac{\langle\mathcal{E}\rangle_{\mathrm{HF}}}{U_{b f} N}} \\
& \times\left[n_{f}\left(\xi_{\tilde{k}}^{f}\right)+n_{b}\left(\xi_{\tilde{k}}^{b}\right)\right],
\end{aligned}
$$

where $\quad \tilde{k}=\sqrt{2 m_{r}\left(\omega_{G}^{\mathrm{GOR}}-\omega_{\mathrm{BP}}\right) / \chi}=$ $\sqrt{2 m_{r}\langle\mathcal{E}\rangle_{\mathrm{HF}}+2 m_{r} U_{b f} N / \chi}$. We see that the factor $\chi^{-3 / 2}$ 

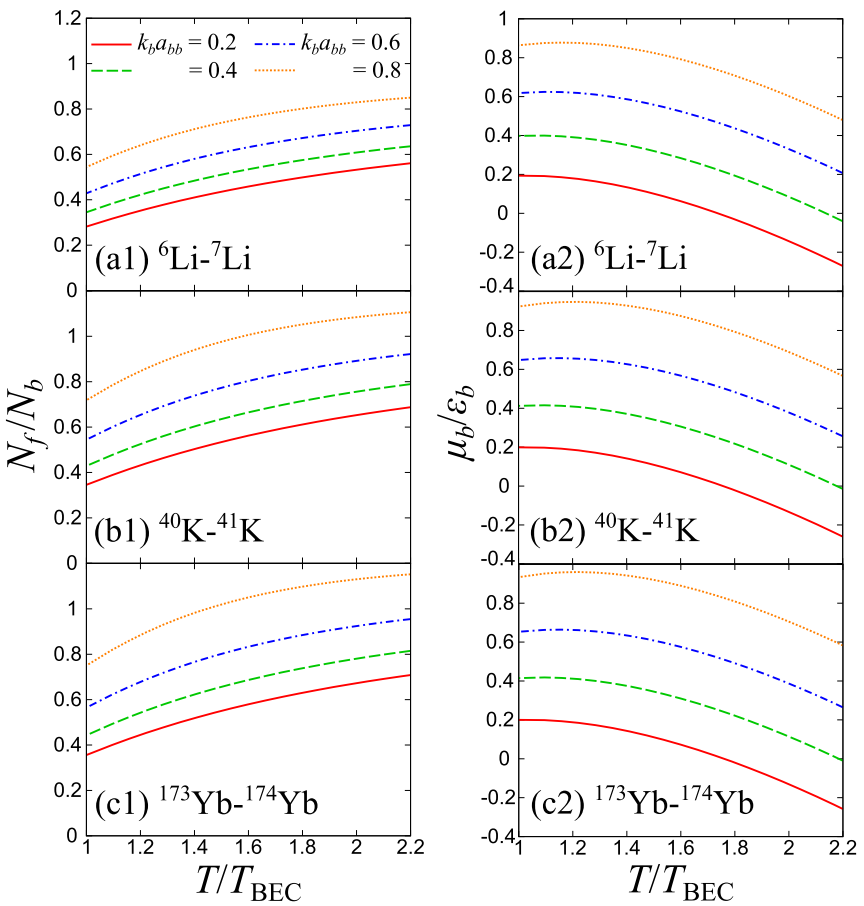

FIG. 2. Ratio between fermionic and bosonic number densities $N_{f} / N_{b}$ with different interactions as functions of the temperature $T$ in (a1) ${ }^{6} \mathrm{Li}-{ }^{7} \mathrm{Li}$, (b1) ${ }^{40} \mathrm{~K}-{ }^{41} \mathrm{~K}$, and (c1) ${ }^{173} \mathrm{Yb}-{ }^{174} \mathrm{Yb}$ Bose-Fermi mixtures with $\Delta \mu=\Delta U=0$. (a2), (b2), and (c2) show the bosonic chemical potential $\mu_{b}$ in systems corresponding to (a1), (b1), and (c1), respectively. In these plots, $\mu_{b}$ is divided by the energy scale $\varepsilon_{b}$ characterizing $N_{b}$ as $\varepsilon_{b}=k_{b}^{2} /\left(2 m_{b}\right)$, where $k_{b}=\left(6 \pi^{2} N_{b}\right)^{\frac{1}{3}}$. $T_{\mathrm{BEC}}$ is the BEC temperature.

appears in contrast to the previous order estimate $\tilde{\Phi} \sim \chi^{2}$. If the $\omega_{G}^{\mathrm{GOR}}$ is far from $\omega_{\mathrm{BP}}$, more precisely, if $\left(\omega_{G}^{\mathrm{GOR}}-\omega_{\mathrm{BP}}\right) \gg \chi T$, the imaginary part is exponentially small by the factor $\exp \left[-\left(\omega_{G}^{\mathrm{GOR}}-\omega_{\mathrm{BP}}\right) /(\chi T)\right]$, so the order estimate $\tilde{\Phi} \sim \chi^{2}$ is still valid.

Since the mass-imbalance effect is generally unavoidable in actual cold-atom experiments, in the following we focus on the mass-imbalanced effect on the gap by taking $\mu_{f}=\mu_{b}$ and $U_{b f}=U_{b b}$, unless otherwise specified. As realistic candidates, we consider ${ }^{6} \mathrm{Li}-{ }^{7} \mathrm{Li},{ }^{40} \mathrm{~K}-{ }^{41} \mathrm{~K}$, and ${ }^{173} \mathrm{Yb}-{ }^{174} \mathrm{Yb}$ mixtures. Even in these systems, it is generally difficult to control $U_{b f}$ and $U_{b b}$ independently. However, in the case of ${ }^{6} \mathrm{Li}-{ }^{7} \mathrm{Li}$ and ${ }^{40} \mathrm{~K}-{ }^{41} \mathrm{~K}$ mixtures, the boson-boson scattering length $a_{b b}=\left(m_{b} U_{b b}\right) /(4 \pi)$ can be tuned due to the magnetic Feshbach resonance [28,29], while the boson-fermion one $a_{b f}=\left(m_{r} U_{b f}\right) /(4 \pi)$ is almost independent of the magnetic field (noting that $a_{b f}=2.16 \mathrm{~nm} \mathrm{[18]} \mathrm{and} a_{b f}=5.13 \mathrm{~nm}$ [20] in ${ }^{6} \mathrm{Li}-{ }^{7} \mathrm{Li}$ and ${ }^{40} \mathrm{~K}-{ }^{41} \mathrm{~K}$ mixtures, respectively). In ${ }^{173} \mathrm{Yb}-{ }^{174} \mathrm{Yb}$ mixtures, two scattering lengths are precisely determined as $a_{b f}=7.34 \mathrm{~nm}$ and $a_{b b}=5.55 \mathrm{~nm}$ [60].

\section{NUMERICAL RESULTS}

\section{A. Thermodynamic quantities}

First, we discuss when the system explicitly breaks the supersymmetry with respect to only the mass imbalance, namely, $U_{b f}=U_{b b}$ and $\mu_{f}=\mu_{b}$ but $m_{b} \neq m_{f}$. Figure 2 shows the fermionic number density $N_{f}$ and the chemical potential $\mu_{b}=\mu_{f}$ for three cases with a fixed bosonic number density $N_{b}$ at which the two conditions above are realized. The BoseEinstein condensation temperature $T_{\mathrm{BEC}}$ is identified by the Hugenholtz-Pines relation [61]

$$
\begin{aligned}
\mu_{b}-\Sigma_{b}= & \mu_{b}-2 U_{b b} N_{b}\left(T=T_{\mathrm{BEC}}\right) \\
& -U_{b f} N_{f}\left(T=T_{\mathrm{BEC}}\right)=0 .
\end{aligned}
$$

We see that $N_{f}$ is smaller than $N_{b}$ at low $T$. The qualitative temperature dependence of these quantities is unchanged among ${ }^{6} \mathrm{Li}-{ }^{7} \mathrm{Li},{ }^{40} \mathrm{~K}-{ }^{41} \mathrm{~K}$, and ${ }^{173} \mathrm{Yb}-{ }^{174} \mathrm{Yb}$ mixtures. While the chemical potential can be precisely obtained through the density profile measurement in recent cold atomic experiments [62-64], it is not in every case. These results on number densities would be helpful for one trying to realize a nearly supersymmetric mixture.

This behavior can be understood as follows: In the noninteracting case, $\mu_{b}=0$ at $T_{\mathrm{BEC}}$ and $\mu_{b}$ is negative above $T_{\mathrm{BEC}}$. In the presence of interactions, the chemical potential is effectively shifted to $\bar{\mu}_{f(b)} \equiv \mu_{f(b)}-\Sigma_{f(b)}^{\mathrm{H}}$ due to the Hartree shift. As $\bar{\mu}_{b}$ is fixed from $N_{b}$ and $T$, which is negative, $\mu_{b}$ becomes larger as the interaction strength increases and eventually becomes positive. On the other hand, $\mu_{f}$ is positive in the low-temperature regime even in the absence of repulsions due to the Fermi-Dirac statistics. Therefore, in the weak-coupling case, $\mu_{f}=\mu_{b}$ would take a positive and small value. As $N_{f}$ is an increasing function of $\bar{\mu}_{f}$, which is proportional to $\mu_{f}, N_{f}$ needs to be much smaller than $N_{b}$ in order to realize $\mu_{f}=\mu_{b}$. This situation is similar to that of so-called Bose polarons [65-71], where impurity atoms (which correspond to fermions in the present case) are immersed in a bosonic medium. If we increase the interaction, $N_{f}$ becomes larger and finally exceeds $N_{b}$.

We note that at stronger coupling, the system may be unstable against phase separation [72]. At $T=0$, the mixture is expected to become unstable when $k_{b} a_{b b} \leqslant \pi \frac{m_{b}}{m_{b}+m_{f}}\left(\frac{N_{b}}{N_{f}}\right)^{\frac{1}{3}}$ at $U_{b b}=U_{b f}$ in Ref. [72]. Since the parameter regimes we consider in this paper are $0.3 \lesssim N_{f} / N_{b} \lesssim 1.2$ (see Fig. 2) and $\frac{7}{13} \leqslant \frac{m_{b}}{m_{f}+m_{b}} \leqslant \frac{174}{347}$, this stability condition can be estimated as $k_{b} a_{b b} \lesssim 1.6$. Furthermore, as usual, such an instability is weakened at finite temperature due to thermal fluctuations. Therefore, although we do not explicitly address this condition, we assume that the homogeneous phase is realized.

\section{B. Spectral properties of the Goldstino}

Using the thermodynamic quantities shown in Fig. 2 and the GOR relation given by Eq. (27) and the RPA equation, (30), we calculate the Goldstino gap above $T_{\mathrm{BEC}}$ as shown in Fig. 3. In the RPA calculation, we have defined $\omega_{G}^{\text {RPA }}$ as the energy where the Goldstino spectral weight $A_{G}(\boldsymbol{p}=\mathbf{0}, \omega)$ has a maximum, where

$$
A_{\mathrm{G}}(\boldsymbol{p}, \omega)=\operatorname{Im} \Gamma^{\mathrm{R}}(\boldsymbol{p}, \omega),
$$

so that it can be defined in the case where the pole has a finite imaginary part. In the case of ${ }^{40} \mathrm{~K}-{ }^{41} \mathrm{~K}$ and ${ }^{173} \mathrm{Yb}-{ }^{174} \mathrm{Yb}$ mixtures at $k_{b} a_{b b}=0.2$, one can find that the GOR predictions show good agreement with the RPA calculation. In fact, one can check that the values of the expansion 


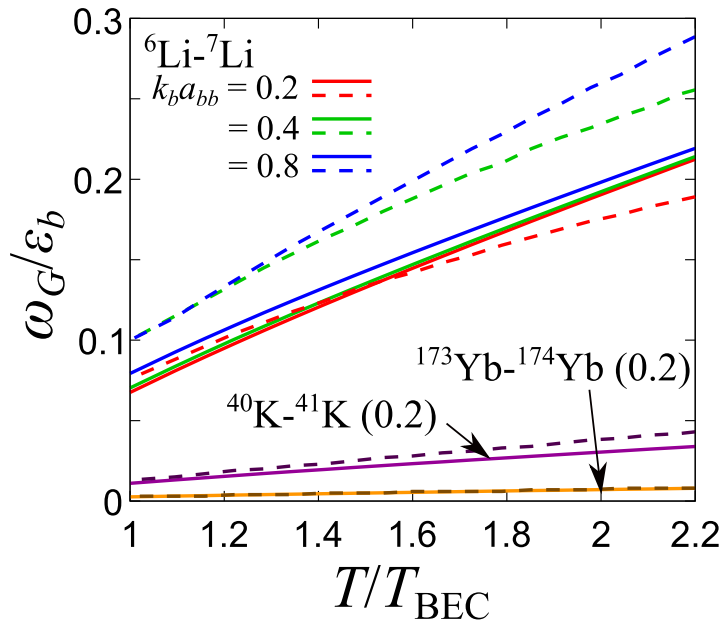

FIG. 3. The Goldstino gap $\omega_{G}$ calculated by the GOR (solid lines) and RPA (dashed lines) with $\Delta U=\Delta \mu=0$. For ${ }^{6} \mathrm{Li}-{ }^{7} \mathrm{Li}$ mixtures, we plot $\omega_{G}$ at different interaction strengths, $k_{b} a_{b b}=0.2$, 0.4 , and 0.8 . We also show $\omega_{G}$ in ${ }^{40} \mathrm{~K}-{ }^{41} \mathrm{~K}$ and ${ }^{173} \mathrm{Yb}-{ }^{174} \mathrm{Yb}$ mixtures at $k_{b} a_{b b}=0.2$.

parameter estimated in Eq. (39) are small: $\left|\omega_{G}^{\mathrm{GOR}} / \omega_{\mathrm{BP}}\right|=$ $9.6 \times 10^{-2}$ and $\left|\omega_{G}^{\mathrm{GOR}} / \omega_{\mathrm{BP}}\right|=2.2 \times 10^{-2}$ in ${ }^{40} \mathrm{~K}-{ }^{-1} \mathrm{~K}$ and ${ }^{173} \mathrm{Yb}-{ }^{174} \mathrm{Yb}$ mixtures, respectively, at $T=T_{\mathrm{BEC}}$. Also, we see that $\omega_{G}^{\mathrm{GOR}} / \varepsilon_{b}$ is quite small. This behavior can be qualitatively understood in the following way: The second term in Eq. (27) gives the term which is proportional to $\chi\langle\mathcal{E}\rangle_{\mathrm{HF}} / \varepsilon_{b}$, in $\omega_{G}^{\mathrm{GOR}} / \varepsilon_{b}$. Assuming that the factor $\langle\mathcal{E}\rangle_{\mathrm{HF}} / \varepsilon_{b}$ is not far from unity, one can make an order estimate of $\omega_{G}^{\mathrm{GOR}} / \varepsilon_{b}$ by checking $\chi$. Indeed, the values $\chi=1.2 \times 10^{-2}$ and $\chi=2.9 \times 10^{-3}$ in ${ }^{40} \mathrm{~K}-{ }^{41} \mathrm{~K}$ and ${ }^{173} \mathrm{Yb}-{ }^{174} \mathrm{Yb}$ mixtures explain the order of magnitude for $\omega_{G}^{\mathrm{GOR}} / \varepsilon_{b}$.

Although we do not show the numerical results explicitly at stronger supersymmetric couplings, this agreement is unchanged in these mixtures. In this regard, we conclude that the mass-imbalance effect in these systems is negligibly small in this temperature region. To confirm the existence of the Goldstino, exploring the interaction and density dependences of the Goldstino gap given by Eq. (29) is suitable. We note that thermodynamic quantities such as the chemical potential were observed precisely within a relative error of less than $4 \%$ in a recent cold-atom experiment [62-64].

On the other hand, the mass-imbalance effect on the Goldstino gap in ${ }^{6} \mathrm{Li}-{ }^{7} \mathrm{Li}$ mixtures with $\chi=1 / 13$ is not so small compared to the other two systems. In fact, the values of the expansion parameter, $\left|\omega_{G}^{\mathrm{GOR}} / \omega_{\mathrm{BP}}\right|=0.621,0.308$, and 0.151 , for $k_{b} a_{b b}=0.2,0.4$, and 0.8 at $T=T_{\mathrm{BEC}}$ are not small compared with those in ${ }^{40} \mathrm{~K}-{ }^{41} \mathrm{~K}$ and ${ }^{173} \mathrm{Yb}-{ }^{174} \mathrm{Yb}$ mixtures. We note that the difference between the GOR and the RPA is accidentally small at $k_{b} a_{b b}=0.2$. This is just a coincidence caused by the singular behavior of the branch point. Figure 4 shows the RPA spectral weight $A_{\mathrm{G}}(\boldsymbol{p}, \omega)$ of the Goldstino at $T=T_{\mathrm{BEC}}, k_{b} a_{b b}=0.2$, and finite momentum. While $A_{\mathrm{G}}(\boldsymbol{p}, \omega)$ in ${ }^{40} \mathrm{~K}-{ }^{41} \mathrm{~K}$ and ${ }^{173} \mathrm{Yb}-{ }^{174} \mathrm{Yb}$ mixtures exhibits a sharp peak associated with the supersymmetric collective mode around $\omega=0$, this peak in a ${ }^{6} \mathrm{Li}-{ }^{7} \mathrm{Li}$ mixture is greatly broadened due to the branch point at weak coupling. We note that the Goldstino spectrum merges with the continuum at

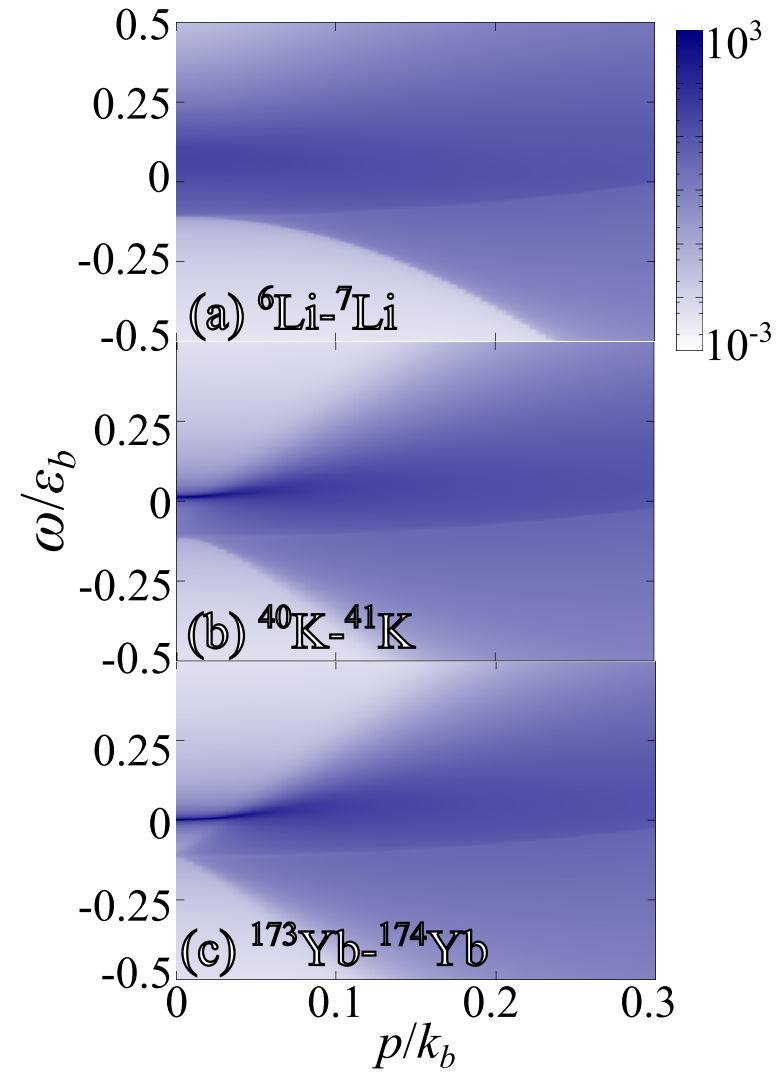

FIG. 4. Calculated Goldstino spectral weight $A_{G}(\boldsymbol{p}, \omega) \varepsilon_{b} / N_{b}$ within the RPA in (a) ${ }^{6} \mathrm{Li}-{ }^{7} \mathrm{Li}$, (b) ${ }^{40} \mathrm{~K}-{ }^{41} \mathrm{~K}$, and (c) ${ }^{173} \mathrm{Yb}-{ }^{174} \mathrm{Yb}$ Bose-Fermi mixtures at $T=T_{\mathrm{BEC}}$. Parameters are set at $k_{b} a_{b b}=0.2$ and $\Delta U=\Delta \mu=0$.

finite momenta even in ${ }^{40} \mathrm{~K}-{ }^{41} \mathrm{~K}$ and ${ }^{173} \mathrm{Yb}-{ }^{174} \mathrm{Yb}$ mixtures. Figure 5(a) shows $A_{G}(\boldsymbol{p}=\mathbf{0}, \omega)$ at zero momentum in a ${ }^{6} \mathrm{Li}-{ }^{7} \mathrm{Li}$ mixture at $T=1.16 T_{\mathrm{BEC}}$. With increasing supersymmetric interaction $k_{b} a_{b b}\left(=\frac{m_{b}}{2 m_{r}} a_{b f}\right.$ since we take $\left.U_{b b}=U_{b f}\right)$, one can see the crossover from the regime where the singularity associated with $\omega_{\mathrm{BP}}$ is dominant to the coexistence of the sharp Goldstino pole and continuum plateau. It is possible to check the sharp Goldstino peak also at finite momentum, from the spectral function at $k_{b} a_{b b}=0.8$ plotted in Fig. 5(b). While in the noninteracting case $\left(k_{b} a_{b b}=0\right)$ a kink structure can be found around $\omega=0$, it originates mainly from the tip of the continuum, as one can see in Appendix B. We note that the contribution at $\omega<0$ in the noninteracting case is an artifact associated with the small imaginary part $i \delta=10^{-3} \varepsilon_{b} i$. We also note that some of the analysis above in a tight-binding model was done in Ref. [13], but this is the first time that we got results for gases of realistic Bose-Fermi mixtures. Figure 6(a) shows a comparison of the Goldstino gap from the GOR relation vs the RPA calculation (corresponding to Fig. 5) in a ${ }^{6} \mathrm{Li}-{ }^{7} \mathrm{Li}$ mixture at $T=1.16 T_{\mathrm{BEC}}$. If we increase $k_{b} a_{b b}$ (and simultaneously $k_{b} a_{b f}$ such that $U_{b f}=U_{b b}$ ), one finds that the two results approach each other around $k_{b} a_{b b} \gtrsim 0.6$. In this regime, as shown in Fig. 5, a sharp Goldstino peak appears since the branch point is separated from the pole. We also plot $\tilde{\Phi}\left(\omega_{G}^{\mathrm{GOR}}\right) / \omega_{G}^{\mathrm{GOR}}$ given by Eq. (37) in Fig. 6(b). Since $\tilde{\Phi}\left(\omega_{G}^{\mathrm{GOR}}\right)$ represents the higher-order corrections included in 

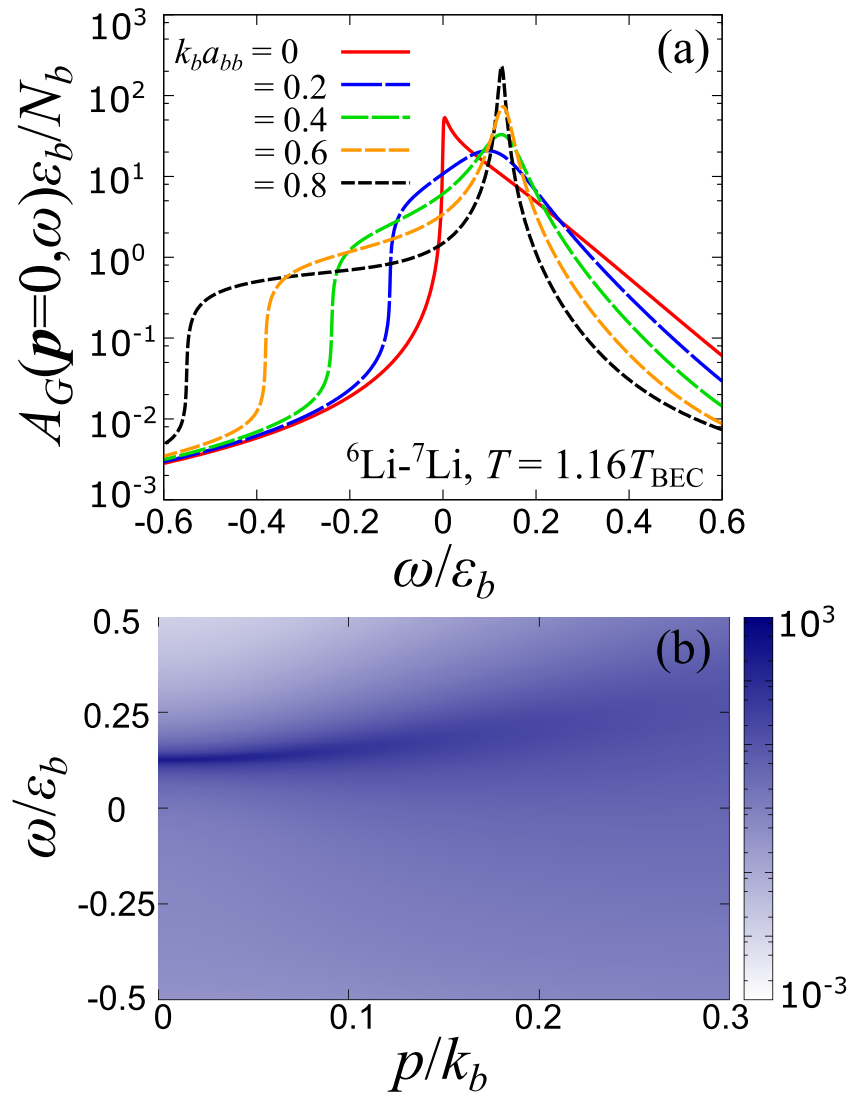

FIG. 5. (a) Goldstino spectral weight $A_{G}(\boldsymbol{p}=\mathbf{0}, \omega)$ at zero momentum in a ${ }^{6} \mathrm{Li}-{ }^{7} \mathrm{Li}$ mixture at $T=1.16 T_{\mathrm{BEC}}$ and $\Delta \mu=\Delta U=0$ with $k_{b} a_{b b}=0,0.2,0.4,0.6$, and 0.8. (b) Contour plot of $A_{G}(\boldsymbol{p}, \omega)$ at $k_{b} a_{b b}=0.8$.

the RPA, the GOR relation is expected to be valid in the region where $\tilde{\Phi}\left(\omega_{G}^{\mathrm{GOR}}\right) / \omega_{G}^{\mathrm{GOR}}$ is small. In this sense, one can obtain the strong intensity of the Goldstino pole even in ${ }^{6} \mathrm{Li}-{ }^{7} \mathrm{Li}$ mixtures in the presence of relatively strong interactions. Such a condition in this case can be expressed as $\chi\langle\mathcal{E}\rangle \ll U_{b f} N$. From Fig. 6(b), one can confirm that the coincidence of $\omega_{G}^{\mathrm{GOR}}$ and $\omega_{G}^{\mathrm{RPA}}$ at $k_{b} a_{b b} \simeq 0.2$ in Figs. 3 and $6(\mathrm{a})$ is accidental due to $\operatorname{Re} \widetilde{\Phi}\left(\omega_{G}^{\mathrm{GOR}}\right)=0$. We note that the Lee-Huang-Yang corrections may become important in the relatively strong repulsive regime [73]. Indeed, this leading quantum correction on $\mu_{b} / \varepsilon_{b}$ is known to be proportional to $\left(k_{b} a_{b b}\right)^{\frac{5}{2}}$ in a single-component Bose gas at $T=0$. A self-consistent framework beyond the present mean-field approximation would be required to obtain the Goldstino gap quantitatively at $k_{b} a_{b b} \gtrsim 1$.

Furthermore, in Fig. 7 we plot $A_{G}(\mathbf{0}, \omega)$ in a ${ }^{173} \mathrm{Yb}-{ }^{174} \mathrm{Yb}$ mixture with realistic interactions given by $a_{b f} / a_{b b}=$ 7.34/5.55 [60], at $T=T_{\mathrm{BEC}}$ and $\mu_{f}=\mu_{b}$. A sharp peak in the Goldstino pole emerges at a positive energy, whereas there is a small peak in the continuum in the negative-energy region. The continuum is quite small compared to the case of the ${ }^{6} \mathrm{Li}-{ }^{7} \mathrm{Li}$ mixture shown in Fig. 5. We have checked that the GOR relation shows excellent agreement with the pole position in this case. If we increase $N_{b}$ [namely, the coupling parameter $k_{b} a_{b b}=\left(6 \pi^{2} N_{b}\right)^{\frac{1}{3}} a_{b b}$ with fixed $a_{b b}$ ], the Goldstino pole becomes distinct since the continuum goes to the lower-

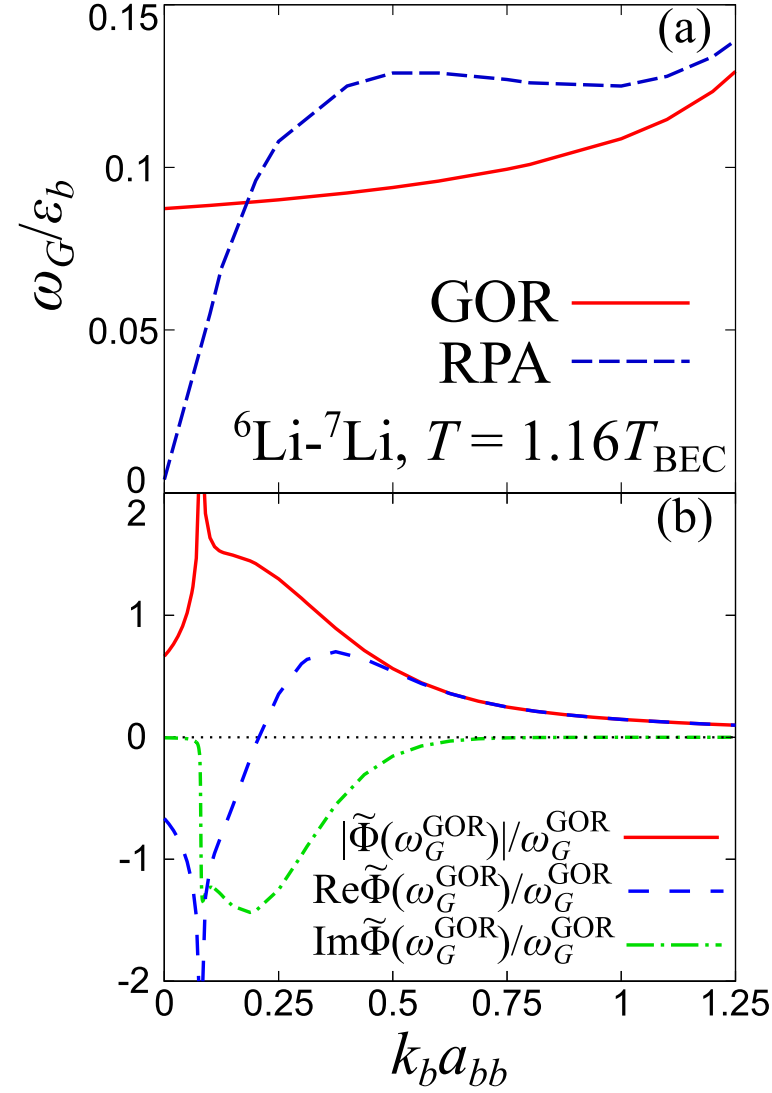

FIG. 6. (a) Comparison of the Goldstino gap $\omega_{G}$ between the GOR and the RPA and (b) $\tilde{\Phi}\left(\omega_{G}^{\mathrm{GOR}}\right) / \omega_{G}^{\mathrm{GOR}}$ given by Eq. (37) in a ${ }^{6} \mathrm{Li}-{ }^{7} \mathrm{Li}$ mixture at $T=1.16 T_{\mathrm{BEC}}$ and $\Delta \mu=\Delta U=0$.

energy region. This result indicates that observation of the Goldstino gap in ${ }^{173} \mathrm{Yb}-{ }^{174} \mathrm{Yb}$ mixtures is quite promising.

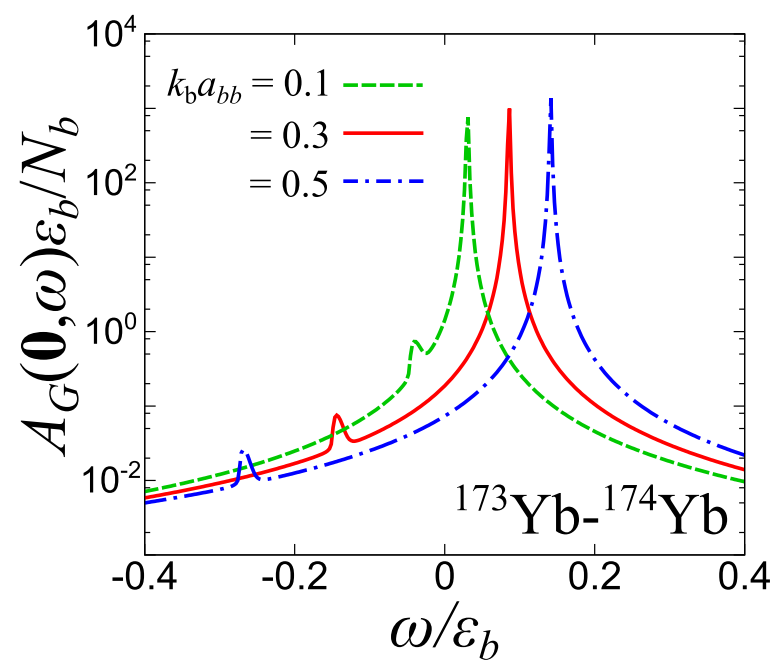

FIG. 7. Calculated Goldstino spectral weight $A_{G}(\boldsymbol{p}=\mathbf{0}, \omega) \varepsilon_{b} / N_{b}$ within the RPA in a ${ }^{173} \mathrm{Yb}-{ }^{174} \mathrm{Yb}$ Bose-Fermi mixture at $T=T_{\mathrm{BEC}}$ and $\Delta \mu=0$. Interaction parameters are chosen to reproduce the experimental ratio $a_{b f} / a_{b b}=7.34 / 5.55$ [60]. The sharp peaks at positive energy are the Goldstino poles. 


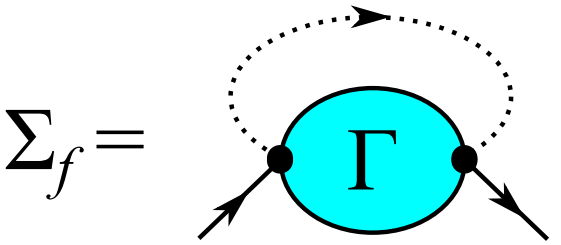

FIG. 8. Self-energy diagram for supersymmetric fluctuations associated with the RPA Goldstino propagator $\Gamma$. Solid (dashed) lines represent fermion (boson) propagators $G_{b(f)}^{\mathrm{H}}$ with the Hartree shift $\Sigma_{f(b)}^{\mathrm{H}}$. Black dots show the boson-fermion coupling $U_{b f}$.

\section{FERMIONIC SINGLE-PARTICLE SPECTRUM}

In this section, in addition to the Tan's contacts shown in Sec. II, we discuss how to detect the Goldstino gap in cold-atom experiments. One promising way is via the singleparticle excitation spectrum of a fermion as discussed in the BEC phase [15]. In the normal phase, we consider the selfenergy $\Sigma_{f}\left(\boldsymbol{p}, i \omega_{\ell}\right)$ diagrammatically drawn in Fig. 8, where $\omega_{\ell}=(2 \ell+1) \pi T$ is the Matsubara frequency of fermions. The explicit form of $\Sigma_{f}\left(\boldsymbol{p}, i \omega_{\ell}\right)$ is given by

$$
\begin{aligned}
\Sigma_{f}\left(\boldsymbol{p}, i \omega_{\ell}\right)= & -U_{b f}^{2} T \sum_{n=-\infty}^{\infty} \int \frac{d^{3} \boldsymbol{k}}{(2 \pi)^{3}} \Gamma\left(\boldsymbol{k}, i \omega_{n}\right) \\
& \times G_{b}^{\mathrm{H}}\left(\boldsymbol{p}-\boldsymbol{k}, i \omega_{\ell}-i \omega_{n}\right),
\end{aligned}
$$

where $G_{b}^{\mathrm{H}}\left(\boldsymbol{p}, i \tilde{\omega}_{k}\right)=1 /\left(i \tilde{\omega}_{k}-\xi_{p}^{b}\right)$ is the Hartree Green's function of a boson. Here, $\tilde{\omega}_{k}=2 k \pi T$ is the Matsubara frequency for bosons. We obtain the single-particle Green's function $G_{f}\left(\boldsymbol{p}, i \omega_{\ell}\right)$ as

$$
G_{f}\left(\boldsymbol{p}, i \omega_{\ell}\right)=\frac{1}{i \omega_{\ell}-\xi_{\boldsymbol{p}}^{f}-\Sigma_{f}\left(\boldsymbol{p}, i \omega_{\ell}\right)} .
$$

The fermionic single-particle spectral function is obtained as $A_{f}(\boldsymbol{p}, \omega)=-\frac{1}{\pi} \operatorname{Im} G_{f}\left(\boldsymbol{p}, i \omega_{\ell} \rightarrow \omega+i \delta\right)$.

Figure 9 shows the calculated $A_{f}(\boldsymbol{p} \rightarrow \mathbf{0}, \omega)$ in a ${ }^{173} \mathrm{Yb}-{ }^{174} \mathrm{Yb}$ mixture at $T=T_{\mathrm{BEC}}$ with realistic interactions, that is, $k_{b} a_{b b}=0.3$ and $a_{b f} / a_{b b}=7.34 / 5.55$ [60]. One finds that $A_{f}(\boldsymbol{p} \rightarrow \mathbf{0}, \omega)$ contains a double-peak structure due to the self-energy correction. In the absence of such a correction, only a single fermionic pole is located at $\omega=\xi_{\boldsymbol{p} \rightarrow \mathbf{0}}^{f} \equiv$ $-\left(\mu_{f}-U_{b f} N_{b}\right)$. In the numerical calculation, we take a finite momentum cutoff, $\Lambda=2 k_{b}$, in Eq. (43). We note that the double-peak structure in $A_{f}(\boldsymbol{p} \rightarrow \mathbf{0}, \omega)$ is left qualitatively unchanged by the value of $\Lambda$ (see also Appendix $C$ ). We examine the qualitative structure of $A_{f}(\boldsymbol{p}, \omega)$ by focusing on the Goldstino pole and using an approximate form of $\Gamma$ as

$$
\Gamma\left(\boldsymbol{k}, i \omega_{n}\right) \simeq \frac{Z_{G}}{i \omega_{n}-E_{\boldsymbol{k}}}
$$

where $E_{k}=k^{2} /\left(2 m_{G}\right)+\omega_{G}$ is the Goldstino dispersion. $Z_{G}$ and $m_{G}$ are the wave-function renormalization and the effective mass of the Goldstino, respectively. Their analytical expressions are obtained in the supersymmetric case at $T=$ 0 [15]. By using this expression, we can analytically perform the summation of the fermion Matsubara frequency in

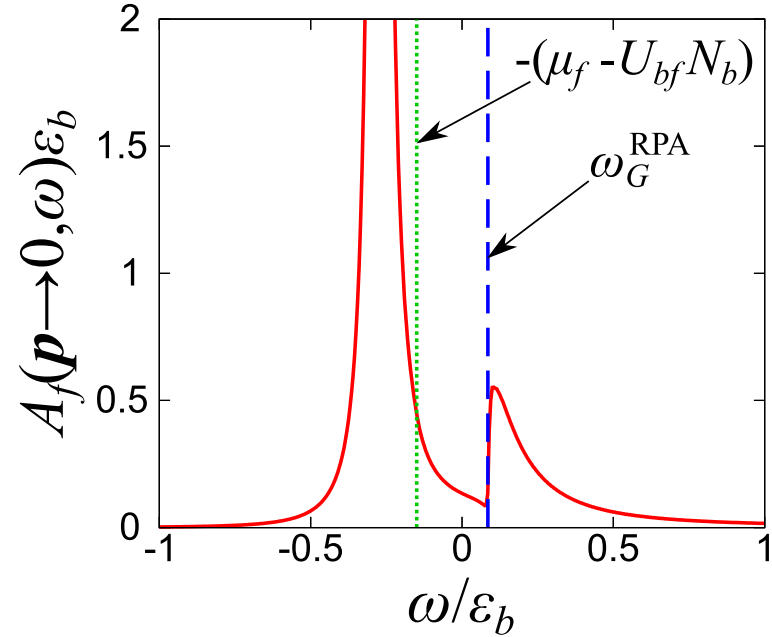

FIG. 9. Fermionic single-particle spectral function $A_{f}(\boldsymbol{p} \rightarrow$ $\mathbf{0}, \omega)$ at the zero-momentum limit in a ${ }^{173} \mathrm{Yb}-{ }^{174} \mathrm{Yb}$ mixture at $T=T_{\mathrm{BEC}}$ with $k_{b} a_{b b}=0.3$ and $a_{b f} / a_{b b}=7.34 / 5.55$ [60]. In the numerical calculation, we take the momentum cutoff $\Lambda=2 k_{b}$. Dotted and dashed vertical lines represent the fermionic pole position $\xi_{p \rightarrow \mathbf{0}}^{f}=-\left(\mu_{f}-U_{b f} N_{b}\right)$ within the mean-field theory and the Goldstino gap $\omega_{G}^{\mathrm{RPA}}$ obtained from the RPA analysis.

$\Sigma_{f}\left(\boldsymbol{p}, i \omega_{\ell}\right)$ as

$\Sigma_{f}\left(\boldsymbol{p}, i \omega_{\ell}\right)=U_{b f}^{2} Z_{G} \int \frac{d^{3} \boldsymbol{k}}{(2 \pi)^{3}} \frac{1-n_{f}\left(E_{\boldsymbol{p}-\boldsymbol{k}}\right)+n_{b}\left(\xi_{\boldsymbol{k}}^{b}\right)}{i \omega_{\ell}-E_{\boldsymbol{p}-\boldsymbol{k}}-\xi_{\boldsymbol{k}}^{b}}$.

Furthermore, near $T=T_{\mathrm{BEC}}$ it was suggested that one can use the so-called static approximation where $n_{b}\left(\xi_{k}^{b}\right)$ has a dominant contribution at $\xi_{k}^{b}=0$ [74]. For qualitative illustrative purposes, we use this approximation and obtain

$$
\Sigma_{f}\left(\boldsymbol{p}, i \omega_{\ell}\right) \simeq \frac{U_{b f}^{2} Z_{G} N_{b}}{i \omega_{\ell}-E_{\boldsymbol{p}}} .
$$

Finally, the fermionic spectral function $A_{f}(\boldsymbol{p} \rightarrow \mathbf{0}, \omega)$ at the zero-momentum limit reads

$$
A_{f}(\boldsymbol{p} \rightarrow \mathbf{0}, \omega)=\alpha_{+} \delta\left(\omega-E_{+}\right)+\alpha_{-} \delta\left(\omega-E_{-}\right),
$$

where

$$
\begin{aligned}
E_{ \pm} & =\frac{\omega_{G}-\mu_{f}+U_{b f} N_{b}}{2} \\
& \pm \sqrt{\left(\frac{\omega_{G}+\mu_{f}-U_{b f} N_{b}}{2}\right)^{2}+U_{b f}^{2} Z_{G} N_{b}}
\end{aligned}
$$

and

$$
\alpha_{ \pm}=\frac{1}{2}\left(1 \mp \frac{\omega_{G}+\mu_{f}-U_{b f} N_{b}}{\sqrt{\left(\omega_{G}+\mu_{f}-U_{b f} N_{b}\right)^{2}+4 U_{b f}^{2} Z_{G} N_{b}}}\right) .
$$

This double-peak structure is due to the level repulsion between the one-particle fermion excitation and the Goldstino pole in $A(\boldsymbol{p} \rightarrow \mathbf{0}, \omega)$. This level repulsion enlarges the separation between the fermionic pole $-\left(\mu_{f}-U_{b f} N_{b}\right)$ and the Goldstino pole $\omega_{G}^{\mathrm{RPA}}$. One can estimate $\omega_{G}$ from $E_{ \pm}$and $\alpha_{ \pm}$. Indeed, in cold-atom experiments, radio-frequency spectroscopies are employed to observe single-particle excitations 
[75]. If the interaction and chemical potential dependences of the low-momentum excitation spectra are observed, one can estimate $\omega_{G}$ from them.

We note that, while we have employed the RPA diagram associated with the fermion-boson bubble resummation given in Fig. 8, fermion-boson ladder diagrams with a many-body $T$ matrix may also appear at the same order [76]. Indeed, the latter diagrams play a crucial role in Bose polarons with attractive interspecies interactions [67] and show an ultraviolet divergence (see Appendix C). This beyond-mean-field correction would be important to predict more quantitative features of fermionic single-particle excitations in the presence of the Goldstino. However, since we consider the repulsive interactions, the ladder diagram in our model does not involve a sharp pole, which is associated with two-body bound molecules in the strongly attractive case, as shown in Refs. [57] and [58] for repulsively interacting two-component Fermi gases. Thus, we expect that our results would correctly capture the qualitative structure of $A_{f}(\boldsymbol{p} \rightarrow \mathbf{0}, \omega)$.

\section{SUMMARY}

To summarize, we have theoretically investigated the gapped Goldstino mode in an ultracold atomic Bose-Fermi mixture with explicitly broken supersymmetry. We have shown the gap formula for the Goldstino (GOR relation) by using the memory function formalism. Using this relation, we calculate the Goldstino gap at the first order of the explicit symmetry breaking and compare it with the numerical results obtained within the RPA. We have confirmed that in the absence of a mass imbalance between fermions and bosons, the Goldstino gap obtained by the GOR relation coincides with that in the RPA. We have also discussed the relationship between the derived GOR relation and the Tan's contact. At the current stage, $\mathrm{a}{ }^{173} \mathrm{Yb}-{ }^{174} \mathrm{Yb}$ mixture is the strongest candidate for detecting the Goldstino. Indeed, using experimental values of scattering lengths and mass ratio, we show that the Goldstino pole has a strong intensity in this mixture. While the mass-imbalance effect in ${ }^{40} \mathrm{~K}-{ }^{41} \mathrm{~K}$ and ${ }^{173} \mathrm{Yb}-{ }^{174} \mathrm{Yb}$ mixtures is negligibly small even in the weak-coupling regime, that in a ${ }^{6} \mathrm{Li}-{ }^{7} \mathrm{Li}$ mixture induces broadening of the Goldstino pole due to the singularity around the branch point in fermionboson bubbles through the infinite sum of bubbles in the RPA. However, if we increase the interactions, the Goldstino pole becomes sharp even in this case since the branch point is well separated from the Goldstino pole. Finally, we have discussed the possibility of observing the Goldstino gap from the single-particle excitation of a Fermi atom. We show the qualitative structure of the spectral function near $T=T_{\mathrm{BEC}}$ and predict the modification of the dispersion due to the coupling between the free branch and the Goldstino pole at low momenta.

To further address realistic experimental situations, it is an important problem to investigate the radio-frequency spectra and the momentum-resolved photoemission spectra of the Fermi atom in the present mixtures. In this case, we have to consider the inhomogeneity due to the trap potential. In addition, exploring other approaches to seeing the Goldstino gap, such as nonequilibrium dynamics [77,78], is also an interesting future direction.

\section{ACKNOWLEDGMENTS}

We would like to thank M. Horikoshi for useful discussion. H.T. was supported by a Grant-in-Aid for JSPS fellows (Grant No. 17J03975) and for Scientific Research from the JSPS (Grant No. 18H05406). Y.H. was supported in part by a Japan Society of Promotion of Science (JSPS) Grant-in-Aid for Scientific Research (KAKENHI Grant Nos. 16K17716, 17H06462, and 18H01211).

\section{APPENDIX A: MEMORY FUNCTION FORMALISM}

In this Appendix, we show the detailed derivation of Eq. (11) based on the memory function formalism [40]. This formalism is useful to describe the Langevin dynamics of slow variables such as the hydrodynamic degrees of freedom. We would like to obtain the equation of $\Gamma^{R}(\omega)$ with the form

$$
(\omega+\Omega+i \Phi(\omega)) \Gamma^{R}(\omega)=-N,
$$

where $\Omega$ and $N$ are constants, and $\Phi(\omega)$ is a function of $\omega$. In the language of the memory function formalism, $K(\omega)=$ $i \Omega-\Phi(\omega)$ is called the memory function; $i \Omega$ and $\Phi(\omega)$ are the static and dynamical parts of the memory function. The corresponding generalized Langevin equation reads

$$
\left(\partial_{t}-i \Omega\right) Q(t)+\int_{0}^{t} d t^{\prime} \Phi\left(t-t^{\prime}\right) Q\left(t^{\prime}\right)=R(t),
$$

where we have introduced the noise $R(t)$ that satisfies $\left\langle Q(t) R\left(t^{\prime}\right)\right\rangle=0$. We do not give the explicit relation between Eq. (A1) and Eq. (A2), which can be shown by using the projection operator method $[79,80]$. Equation (A1) has a similar form to the Schwinger-Dyson equation in quantum field theory. Roughly speaking, $\Omega+i \Phi(\omega)$ corresponds to the selfenergy, and $\Omega$ and $\Phi$ give the gap and dissipation, respectively. The purpose here is to express $\Omega, N$, and $\Phi(\omega)$ by correlation functions. For this purpose, we introduce the Liouville operator $\mathcal{L}$ as $\mathcal{L} q \equiv[H, q]$ such that we can express $q(\boldsymbol{r}, t)$ as $q(\boldsymbol{r}, t)=e^{i \mathcal{L} t} q(\boldsymbol{r}, 0)$. This enables us to rewrite Eq. (10) at $\boldsymbol{p}=\mathbf{0}$ as

$$
\Gamma^{R}(\omega)=i\left\langle\left\{r(\omega) Q, q^{\dagger}(\mathbf{0}, 0)\right\}\right\rangle,
$$

where $r(\omega)=-i /(\omega+\mathcal{L})$. Using the identity $-i \omega r(\omega)=$ $1+i \mathcal{L} r(\omega)$, we obtain

$$
-i \omega \Gamma^{R}(\mathbf{0}, \omega)=i N+i\left\langle\left\{r(\omega) i \mathcal{L} Q, q^{\dagger}(\mathbf{0}, 0)\right\}\right\rangle,
$$

where

$$
N=\left\langle\psi_{f}^{\dagger}(\boldsymbol{r}) \psi_{f}(\boldsymbol{r})\right\rangle+\left\langle\psi_{b}^{\dagger}(\boldsymbol{r}) \psi_{b}(\boldsymbol{r})\right\rangle
$$

is the total number density. We now introduce the memory function $K(z)$ such that

$$
K(\omega) \Gamma^{R}(\mathbf{0}, \omega)=i\left\langle\left\{r(\omega) i \mathcal{L} Q, q^{\dagger}(\mathbf{0}, 0)\right\}\right\rangle .
$$

By construction, this satisfies $(\omega-i K(\omega)) \Gamma^{R}(\omega)=-N$. We would further like to decompose $K(\omega)$ into the static part $i \Omega$, which is responsible for the gap, and the dynamic one $\Phi(\omega)$, which is responsible for the dissipation. Multiplying Eq. (A6) 
by $-i \omega$ and using Eq. (A4) and $-i \omega r(\omega)=1+i \mathcal{L} r(\omega)$, we obtain

$$
\begin{aligned}
& K(\omega)\left[i N+i\left\langle\left\{r(\omega) i \mathcal{L} Q, q^{\dagger}(\mathbf{0}, 0)\right\}\right\rangle\right] \\
& \quad=i\left\langle\left\{i \mathcal{L} Q, q^{\dagger}(\mathbf{0}, 0)\right\}\right\rangle+i\left\langle\left\{r(\omega)(i \mathcal{L})^{2} Q, q^{\dagger}(\mathbf{0}, 0)\right\}\right\rangle .
\end{aligned}
$$

From Eq. (A6), the left-hand side of Eq. (A7) can be written as

$$
i N K(\omega)+i \frac{i}{\Gamma^{R}(\mathbf{0}, \omega)}\left\langle\left\{r(z) i \mathcal{L} Q, q^{\dagger}(\mathbf{0}, 0)\right\}\right\rangle^{2} .
$$

Substituting Eq. (A8) into Eq. (A7), we obtain $K(\omega)=$ $i \Omega-\Phi(\omega)$ with

$$
\begin{gathered}
i \Omega=\frac{1}{N}\left\langle\left\{i \mathcal{L} Q, q^{\dagger}(\mathbf{0}, 0)\right\}\right\rangle, \\
\Phi(\omega)=-\frac{1}{N}\left\langle\left\{r(\omega)(i \mathcal{L})^{2} Q, q^{\dagger}(\mathbf{0}, 0)\right\}\right\rangle \\
+\frac{1}{N} \frac{i}{\Gamma^{R}(\mathbf{0}, \omega)}\left\langle\left\{r(\omega) i \mathcal{L} Q, q^{\dagger}(\mathbf{0}, 0)\right\}\right\rangle^{2} .
\end{gathered}
$$

Noting the relation

$$
\begin{aligned}
\langle\{i \mathcal{L} A, B\}\rangle & =\frac{i}{\operatorname{tr} e^{-\beta H}} \operatorname{tr} e^{-\beta H}\{[H, A], B\} \\
& =\frac{-i}{\operatorname{tr} e^{-\beta H}} \operatorname{tr} e^{-\beta H}\{A,[H, B]\}=-\langle\{A, i \mathcal{L} B\}\rangle,
\end{aligned}
$$

we can express the dynamic part as

$$
\begin{aligned}
\Phi(\omega)= & \frac{1}{N}\left\langle\left\{r(\omega) i \mathcal{L} Q, i \mathcal{L} q^{\dagger}(\mathbf{0}, 0)\right\}\right\rangle \\
& +\frac{1}{N}\left\langle\left\{r(\omega) i \mathcal{L} Q, q^{\dagger}(\mathbf{0}, 0)\right\}\right\rangle \\
& \times \frac{i}{\Gamma^{R}(\mathbf{0}, \omega)}\left\langle\left\{r(\omega) Q, i \mathcal{L} q^{\dagger}(\mathbf{0}, 0)\right\}\right\rangle .
\end{aligned}
$$

In summary, the retarded Green function satisfies Eq. (A1), which corresponds to the generalized Langevin equation, (A2). The coefficients and function are given by Eqs. (A5), (A9), and (A12).

Let us check the order of $\Omega$ and $\Phi(\omega)$ with respect to the explicit breaking term. Since both static and dynamic parts are proportional to $i \mathcal{L} Q=i[H, Q], K(\omega)$ vanishes if the supersymmetry is exact. Therefore, $\omega=0$ becomes the pole. When the supersymmetry is explicitly broken by a small parameter, $[H, Q] \sim \epsilon$, the static part is $i \Omega \sim \epsilon$, while the dynamic part is $\Phi(\omega) \sim \epsilon^{2}$ as shown in Eq. (A12). Therefore, at leading order in $\epsilon$, we can neglect $\Phi(\omega)$. We note that we also assigned $\left[H, q^{\dagger}(\boldsymbol{x}, t)\right] \sim \epsilon$. Precisely speaking, there is the other contribution of order 1 , the divergence of the supersymmetric current $\nabla \cdot \boldsymbol{j}$ in $\left[H, q^{\dagger}(\boldsymbol{x}, t)\right]$. This vanishes in the correlation function at $\boldsymbol{p}=\mathbf{0}$. At leading order in $\epsilon$, we find the pole, (12).

\section{APPENDIX B: ANALYTICAL RESULTS IN A NONINTERACTING MIXTURE}

To check that the numerical procedure to include the infinitesimal imaginary part $i \delta$ in the analytic continuation does not cause a serious numerical artifact, we investigate the Goldstino spectral function in the free limit. In the noninteracting

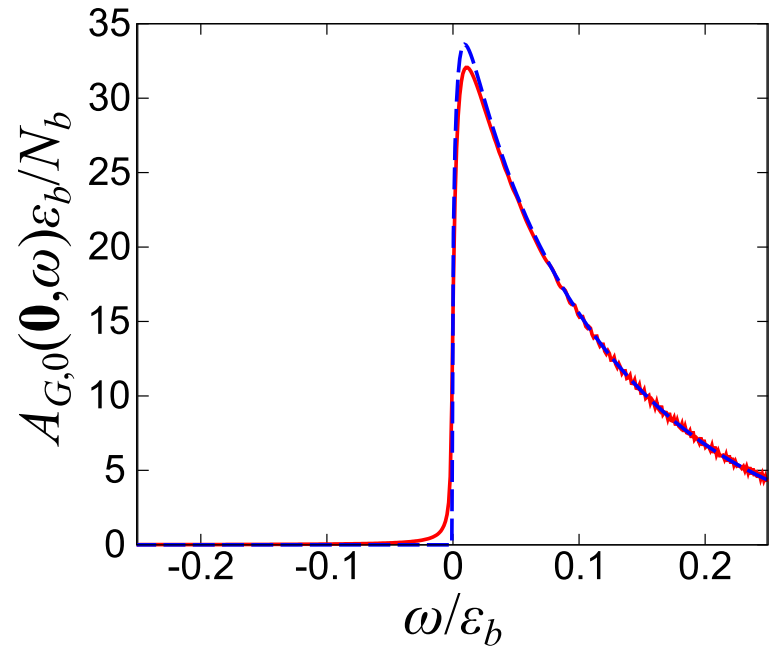

FIG. 10. Comparison of $A_{G, 0}(\mathbf{0}, \omega)$ in a noninteracting ${ }^{6} \mathrm{Li}-{ }^{7} \mathrm{Li}$ mixture at $T=1.38 T_{\mathrm{BEC}}$ with $\mu_{b}=\mu_{f}$ obtained from Eq. (B2) (dashed curve) and the numerical result with $\delta=10^{-3} \varepsilon_{b}$ (solid curve).

case, the spectral weight $A_{G, 0}(\boldsymbol{p}=\mathbf{0}, \omega)$ reads

$$
\begin{aligned}
A_{G, 0}(\mathbf{0}, \omega)= & -\operatorname{Im} \int \frac{d^{3} \boldsymbol{k}}{(2 \pi)^{3}} \frac{n_{b}\left(\xi_{\boldsymbol{k}}^{b}\right)+n_{f}\left(\xi_{\boldsymbol{k}}^{f}\right)}{\omega+\Delta \mu+i \delta-\chi k^{2} / 2} \\
= & \int_{0}^{\infty} \frac{k^{2} d k}{2 \pi}\left[n_{b}\left(\xi_{\boldsymbol{k}}^{b}\right)+n_{f}\left(\xi_{\boldsymbol{k}}^{f}\right)\right] \\
& \times \delta\left(\omega+\Delta \mu-\chi \frac{k^{2}}{2}\right) \quad(\delta \rightarrow 0) .
\end{aligned}
$$

By performing momentum integration, we can obtain the analytical expression of $A_{G}(\mathbf{0}, \omega)$ as

$$
\begin{aligned}
A_{G, 0}(\mathbf{0}, \omega)= & \frac{1}{\sqrt{2} \pi \chi^{\frac{3}{2}}} \theta(\omega) \sqrt{\omega} \\
& \times\left[\frac{1}{e^{\left(\frac{\omega}{m_{b}}-\mu_{b}\right) / T}-1}+\frac{1}{e^{\left(\frac{\omega}{m_{f} \chi}-\mu_{f}\right) / T}+1}\right]
\end{aligned}
$$

at $\Delta \mu=0$. If we take $\chi \rightarrow 0, A_{G, 0}(\mathbf{0}, \omega)$ diverges at only $\omega=$ 0 , which indicates that the continuum has vanishing width at zero momentum.

It can be understood also from the sum rule $[14,15]$, $\int d \omega A_{G}(\boldsymbol{p}, \omega) / \pi=N$ : At finite $\omega, A_{G, 0}$ vanishes at $\chi \rightarrow 0$, due to the exponential factor. Therefore, to satisfy the sum rule, the existence of divergence at $\omega=0$ is implied.

In Fig. 10 we show a comparison between Eq. (B2) and the numerical result with $\delta=10^{-3}$ in a noninteracting ${ }^{6} \mathrm{Li}-{ }^{7} \mathrm{Li}$ mixture at $T=1.38 T_{\mathrm{BEC}}$. From this, we find that the effect of $\delta$ is small for the maximum of $A_{G, 0}(\mathbf{0}, \omega)$. While the finite contribution at $\omega<0$ in the numerical calculation originates from the finite $\delta$, we confirmed that this also does not affect our main results. 


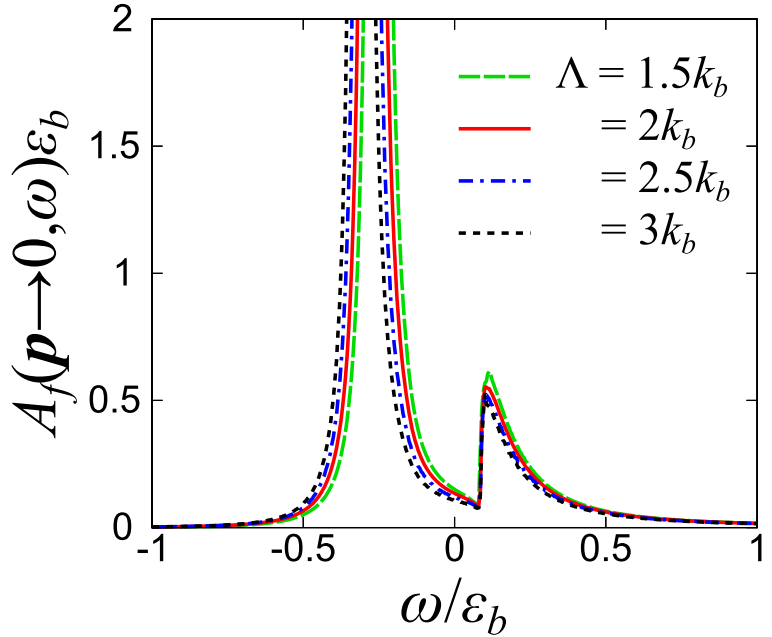

FIG. 11. Fermionic single-particle spectral function $A_{f}(\boldsymbol{p} \rightarrow \mathbf{0}$, $\omega)$ at different momentum cutoffs, $\Lambda / k_{b}=1.5,2,2.5$, and 3. Other parameters are the same as in Fig. 9.

\section{APPENDIX C: ULTRAVIOLET CUTOFF DEPENDENCE OF THE FERMIONIC SINGLE-PARTICLE SPECTRAL FUNCTION}

The fermionic self-energy $\Sigma_{f}\left(\boldsymbol{p}, i \omega_{n} \rightarrow \omega+i \delta\right)$ analytically continued to the real frequency reads

$$
\begin{aligned}
\Sigma_{f}(\boldsymbol{p}, \omega+i \delta)= & U_{b f}^{2} \int^{|\boldsymbol{k}| \leqslant \Lambda} \frac{d^{3} \boldsymbol{k}}{(2 \pi)^{3}} \int_{-\infty}^{\infty} d z A_{G}(\boldsymbol{k}, z) \\
& \times \frac{1-n_{f}(z)+n_{b}\left(\xi_{\boldsymbol{p}-\boldsymbol{k}}^{b}\right)}{\omega+i \delta-z-\xi_{\boldsymbol{p}-\boldsymbol{k}}^{b}}
\end{aligned}
$$

Figure 11 shows the calculated $A_{f}(\boldsymbol{p} \rightarrow 0, \omega)$ with different cutoffs $\Lambda$. One can see that the lower sharp peak is quantitatively shifted toward the lower-energy side with increasing $\Lambda$. We emphasize, however, that the doublepeak structure is qualitatively unchanged by the value of $\Lambda$.

On the other hand, the particle-particle ladder diagram, which is not considered in this work, is known to exhibit an ultraviolet divergence. The many-body $T$ matrix responsible for this scattering process is given by

$$
T_{\mathrm{MB}}\left(\boldsymbol{k}, i \omega_{n}\right)=\frac{U_{b f}}{1+U_{b f} L\left(\boldsymbol{k}, i \omega_{n}\right)},
$$

where

$$
L\left(\boldsymbol{k}, i \omega_{n}\right)=-\int^{|\boldsymbol{p}| \leqslant \Lambda} \frac{d^{3} \boldsymbol{p}}{(2 \pi)^{3}} \frac{1-n_{f}\left(\xi_{\boldsymbol{p}+\boldsymbol{k} / 2}^{f}\right)+n_{b}\left(\xi_{-\boldsymbol{p}+\boldsymbol{k} / 2}^{b}\right)}{i \omega_{n}-\xi_{\boldsymbol{p}+\boldsymbol{k} / 2}^{f}-\xi_{-\boldsymbol{p}+\boldsymbol{k} / 2}^{b}}
$$

is the lowest-order particle-particle correlation function. $T_{\mathrm{MB}}\left(\boldsymbol{k}, i \omega_{n}\right)$ and mixing of the ladder and bubble diagrams may appear in the self-energy corrections in addition to Eq. (43). One can see that Eq. (C3) involves an ultraviolet divergence. In this case, the relation between $U_{b f}$ and $a_{b f}$ is replaced by

$$
\frac{m_{r}}{2 \pi a_{b f}}=\frac{1}{U_{b f}}+\frac{m_{r} \Lambda}{\pi^{2}} .
$$

Here, the cutoff $\Lambda$, which is proportional to an inverse effective range [58], should be finite to reproduce the finite $a_{b f}$. These ladder and finite-range corrections remain as future work for more quantitative descriptions of $A_{f}(\boldsymbol{p}, \omega)$.
[1] P. Fayet and S. Ferrara, Supersymmetry, Phys. Rep. 32, 249 (1977).

[2] E. Witten, Constraints on supersymmetry breaking, Nucl. Phys. B 202, 253 (1982).

[3] H. P. Nilles, Supersymmetry, supergravity and particle physics, Phys. Rep. 110, 1 (1984).

[4] J. L. Feng, J.-F. Grivaz, and J. Nachtman, Searches for supersymmetry at high-energy colliders, Rev. Mod. Phys. 82, 699 (2010).

[5] F. Dalfovo, S. Giorgini, L. P. Pitaevskii, and S. Stringari, Theory of Bose-Einstein condensation in trapped gases, Rev. Mod. Phys. 71, 463 (1999).

[6] S. Giorgini, L. P. Pitaevskii, and S. Stringari, Theory of ultracold atomic Fermi gases, Rev. Mod. Phys. 80, 1215 (2008).

[7] I. Bloch, J. Dalibard, and W. Zwerger, Many-body physics with ultracold gases, Rev. Mod. Phys. 80, 885 (2008).

[8] C. Chin, R. Grimm, P. Julienne, and E. Tiesinga, Feshbach resonances in ultracold gases, Rev. Mod. Phys. 82, 1225 (2010).

[9] M. Snoek, S. Vandoren, and H. T. C. Stoof, Theory of ultracold superstrings, Phys. Rev. A 74, 033607 (2006).

[10] Y. Yu and K. Yang, Supersymmetry and the Goldstino-like mode in Bose-Fermi mixtures, Phys. Rev. Lett. 100, 090404 (2008).
[11] Y. Yu and K. Yang, Simulating the Wess-Zumino Supersymmetry Model in Optical Lattices, Phys. Rev. Lett. 105, 150605 (2010).

[12] H.-H. Lai and K. Yang, Relaxation of a Goldstino-like mode due to supersymmetry breaking in Bose-Fermi mixtures, Phys. Rev. A 91, 063620 (2015).

[13] T. Shi, Y. Yu, and C. P. Sun, Supersymmetric response of a Bose-Fermi mixture to photoassociation, Phys. Rev. A 81, 011604(R) (2010).

[14] J.-P. Blaizot, Y. Hidaka, and D. Satow, Spectral properties of the Goldstino in supersymmetric Bose-Fermi mixtures, Phys. Rev. A 92, 063629 (2015).

[15] J.-P. Blaizot, Y. Hidaka, and D. Satow, Goldstino in supersymmetric Bose-Fermi mixtures in the presence of a Bose-Einstein condensate, Phys. Rev. A 96, 063617 (2017).

[16] B. Bradlyn and A. Gromov, Supersymmetric waves in BoseFermi mixtures, Phys. Rev. A 93, 033642 (2016).

[17] I. Ferrier-Barbut, M. Delehaye, S. Laurent, A. T. Grier, M. Pierce, B. S. Rem, F. Chevy, and C. Salomon, A mixture of Bose and Fermi superfluids, Science 345, 1035 (2014).

[18] S. Laurent, M. Pierce, M. Delehaye, T. Yefsah, F. Chevy, and C. Salomon, Connecting Few-Body Inelastic Decay to Quantum Correlations in a Many-Body System: A Weakly Coupled 
Impurity in a Resonant Fermi Gas, Phys. Rev. Lett. 118, 103403 (2017).

[19] T. Ikemachi, A. Ito, Y. Aratake, Y. Chen, M. Koashi, M. Kuwata-Gonokami, and M. Horikoshi, All-optical production of dual Bose-Einstein condensates of paired fermions and bosons with ${ }^{6} \mathrm{Li}$ and ${ }^{7} \mathrm{Li}$, J. Phys. B: At. Mol. Opt. Phys. 50, 01LT01 (2017).

[20] S. Falke, H. Knöckel, J. Friebe, M. Riedmann, E. Tiemann, and C. Lisdat, Potassium ground-state scattering parameters and Born-Oppenheimer potentials from molecular spectroscopy, Phys. Rev. A 78, 012503 (2008).

[21] C.-H. Wu, I. Santiago, J. W. Park, P. Ahmadi, and M. W. Zwierlein, Quantum degenerate Bose-Fermi mixture of chemically different atomic species with widely tunable interactions, Phys. Rev. A 84, 011601(R) (2011).

[22] M. K. Tey, S. Stellmer, R. Grimm, and F. Schreck, Doubledegenerate Bose-Fermi mixture of strontium, Phys. Rev. A 82, 011608(R) (2010).

[23] V. Barbé, A. Ciamei, B. Pasquiou, L. Rechsöllner, F. Schreck, P. S. Żuchowski, and J. M. Hutson, Observation of feshbach resonances between alkali and closed-shell atoms, Nat. Phys. 14, 881 (2018).

[24] M. Lu, N. Q. Burdick, and B. L. Lev, Quantum Degenerate Dipolar Fermi Gas, Phys. Rev. Lett. 108, 215301 (2012).

[25] T. Fukuhara, S. Sugawa, Y. Takasu, and Y. Takahashi, Alloptical formation of quantum degenerate mixtures, Phys. Rev. A 79, 021601(R) (2009).

[26] S. Sugawa, K. Inaba, S. Taie, R. Yamazaki, M. Yamashita, and Y. Takahashi, Interaction and filling-induced quantum phases of dual Mott insulators of bosons and fermions, Nat. Phys. 7, 642 (2011).

[27] C. D’Errico, M. Zaccanti, M. Fattori, G. Roati, M. Inguscio, G. Modugno, and A. Simoni, Feshbach resonances in ultracold ${ }^{39}$ K, New J. Phys. 9, 223 (2007).

[28] S. E. Pollack, D. Dries, M. Junker, Y. P. Chen, T. A. Corcovilos, and R. G. Hulet, Extreme tunability of interactions in a ${ }^{7}$ Li Bose-Einstein condensate, Phys. Rev. Lett. 102, 090402 (2009).

[29] T. Kishimoto, J. Kobayashi, K. Noda, K. Aikawa, M. Ueda, and $\mathrm{S}$. Inouye, Direct evaporative cooling of ${ }^{41} \mathrm{~K}$ into a BoseEinstein condensate, Phys. Rev. A 79, 031602(R) (2009).

[30] A. Salam and J. Strathdee, On goldstone fermions, Phys. Lett. B 49, 465 (1974).

[31] E. Witten, Dynamical breaking of supersymmetry, Nucl. Phys. B 188, 513 (1981).

[32] V. V. Lebedev and A. V. Smilga, Supersymmetric sound, Nucl. Phys. B 318, 669 (1989).

[33] K. Kratzert, Finite temperature supersymmetry: The Wess-Zumino model, Ann. Phys. 308, 285 (2003).

[34] K. Kratzert, Supersymmetry breaking at finite temperature, Ph.D. thesis, University of Hamburg, 2002.

[35] V. V. Lebedev and A. V. Smilga, Spectrum of quark-gluon plasma, Ann. Phys. 202, 229 (1990).

[36] Y. Hidaka, D. Satow, and T. Kunihiro, Ultrasoft fermionic modes at high temperature, Nucl. Phys. A 876, 93 (2012).

[37] D. Satow, Ultrasoft fermion mode and off-diagonal Boltzmann equation in a quark-gluon plasma at high temperature, Phys. Rev. D 87, 096011 (2013).

[38] T. Hatsuda and T. Kunihiro, QCD phenomenology based on a chiral effective Lagrangian, Phys. Rep. 247, 221 (1994).
[39] M. Gell-Mann, R. Oakes, and B. Renner, Behavior of current divergences under $\mathrm{SU}_{3} \times \mathrm{SU}_{3}$, Phys. Rev. 175, 2195 (1968).

[40] G. F. Mazenko, Nonequilibrium Statistical Mechanics (WileyVCH, Weinheim, 2006).

[41] J. Zhang, E. G. M. van Kempen, T. Bourdel, L. Khaykovich, J. Cubizolles, F. Chevy, M. Teichmann, L. Tarruell, S. J. J. M. F. Kokkelmans, and C. Salomon, $P$-wave Feshbach resonances of ultracold ${ }^{6}$ Li, Phys. Rev. A 70, 030702(R) (2004).

[42] S. Pilati, K. Sakkos, J. Boronat, J. Casulleras, and S. Giorgini, Equation of state of an interacting Bose gas at finite temperature: A path-integral Monte Carlo study, Phys. Rev. A 74, 043621 (2006).

[43] A. L. Fetter and J. D. Walecka, Quantum Theory of ManyParticle Systems (Dover, London, 1971).

[44] H. Watanabe and T. Brauner, Number of Nambu-Goldstone bosons and its relation to charge densities, Phys. Rev. D 84, 125013 (2011).

[45] H. Watanabe and H. Murayama, Unified Description of NambuGoldstone Bosons without Lorentz Invariance, Phys. Rev. Lett. 108, 251602 (2012).

[46] Y. Hidaka, Counting Rule for Nambu-Goldstone Modes in Nonrelativistic Systems, Phys. Rev. Lett. 110, 091601 (2013).

[47] H. Watanabe and H. Murayama, Effective Lagrangian for Nonrelativistic Systems, Phys. Rev. X 4, 031057 (2014).

[48] T. Hayata and Y. Hidaka, Dispersion relations of NambuGoldstone modes at finite temperature and density, Phys. Rev. D 91, 056006 (2015).

[49] D. A. Takahashi and M. Nitta, Counting rule of NambuGoldstone modes for internal and spacetime symmetries: Bogoliubov theory approach, Ann. Phys. 354, 101 (2015).

[50] S. Tan, Energetics of a strongly correlated Fermi gas, Ann. Phys. 323, 2952 (2008).

[51] S. Tan, Large momentum part of a strongly correlated Fermi gas, Ann. Phys. 323, 2971 (2008).

[52] S. Tan, Generalized virial theorem and pressure relation for a strongly correlated Fermi gas, Ann. Phys. 323, 2987 (2008).

[53] E. Braaten, D. Kang, and L. Platter, Universal relations for a strongly interacting Fermi gas near a Feshbach resonance, Phys. Rev. A 78, 053606 (2008).

[54] J. M. Diederix, T. C. F. van Heijst, and H. T. C. Stoof, Ground state of a resonantly interacting Bose gas, Phys. Rev. A 84, 033618 (2011).

[55] C. Qu, L. P. Pitaevskii, and S. Stringari, Magnetic solitons in Rabi-coupled Bose-Einstein condensates, Phys. Rev. A 94, 063635 (2016).

[56] X.-J. Liu, B. Mulkerin, L. He, and H. Hu, Equation of state and contact of a strongly interacting Bose gas in the normal state, Phys. Rev. A 91, 043631 (2015).

[57] W. Schneider and M. Randeria, Universal short-distance structure of the single-particle spectral function of dilute Fermi gases, Phys. Rev. A 81, 021601(R) (2010).

[58] F. Palestini, A. Perali, P. Pieri, and G. C. Strinati, Dispersions, weights, and widths of the single-particle spectral function in the normal phase of a Fermi gas, Phys. Rev. B 85, 024517 (2012).

[59] J. O. Andersen, Theory of the weakly interacting Bose gas, Rev. Mod. Phys. 76, 599 (2004).

[60] M. Kitagawa, K. Enomoto, K. Kasa, Y. Takahashi, R. Ciuryło, P. Naidon, and P. S. Julienne, Two-color photoassociation spectroscopy of ytterbium atoms and the precise determina- 
tions of $s$-wave scattering lengths, Phys. Rev. A 77, 012719 (2008).

[61] N. M. Hugenholtz and D. Pines, Ground-state energy and excitation spectrum of a system of interacting bosons, Phys. Rev. 116, 489 (1959).

[62] M. Horikoshi, M. Koashi, H. Tajima, Y. Ohashi, and M. Kuwata-Gonokami, Ground-State Thermodynamic Quantities of Homogeneous Spin-1/2 Fermions from the BCS Region to the Unitarity Limit, Phys. Rev. X 7, 041004 (2017).

[63] H. Tajima, P. van Wyk, R. Hanai, D. Kagamihara, D. Inotani, and M. Horikoshi, and Y. Ohashi, Strong-coupling corrections to ground-state properties of a superfluid Fermi gas, Phys. Rev. A 95, 043625 (2017).

[64] M. Horikoshi and M. Kuwata-Gonokami, Cold atom quantum simulator for dilute neutron matter, Int. J. Mod. Phys. E 28, 1930001 (2019).

[65] F. M. Cucchietti and E. Timmermans, Strong-Coupling Polarons in Dilute Gas Bose-Einstein Condensates, Phys. Rev. Lett. 96, 210401 (2006).

[66] D. H. Santamore and E. Timmermans, Multi-impurity polarons in a dilute Bose-Einstein condensate, New J. Phys. 13, 103029 (2011).

[67] S. P. Rath and R. Schmidt, Field-theoretical study of the Bose polaron, Phys. Rev. A 88, 053632 (2013).

[68] W. Li and S. D. Sarma, Variational study of polarons in BoseEinstein condensates, Phys. Rev. A 90, 013618 (2014).

[69] M.-G. Hu, M. J. Van de Graaff, D. Kedar, J. P. Corson, E. A. Cornell, and D. S. Jin, Bose Polarons in the Strongly Interacting Regime, Phys. Rev. Lett. 117, 055301 (2016).

[70] J. Takahashi, R. Imai, E. Nakano, and K. Iida, Bose polaron in spherical trap potentials: Spatial structure and quantum depletion, Phys. Rev. A 100, 023624 (2019).
[71] S. I. Mistakidis, L. Hilbig, and P. Schmelcher, Correlated quantum dynamics of two quenched fermionic impurities immersed in a Bose-Einstein condensate, Phys. Rev. A 100, 023620 (2019).

[72] L. Viverit, C. J. Pethick, and H. Smith, Zero-temperature phase diagram of binary boson-fermion mixtures, Phys. Rev. A 61, 053605 (2000).

[73] T. D. Lee, K. Huang, and C. N. Yang, Eigenvalues and eigenfunctions of a Bose system of Hard spheres and its low-temperature properties, Phys. Rev. 106, 1135 (1957).

[74] D. Kharga, D. Inotani, R. Hanai, and Y. Ohashi, Single-particle excitations and effects of hetero-pairing fluctuations in a Bose-Fermi mixture with a Feshbach resonance, J. Phys. Soc. Jpn. 86, 084301 (2017).

[75] P. Törmä, Spectroscopies-Theory, in Quantum Gas Experiments-Exploring Many-Body States, edited by $\mathrm{P}$. Törmä and K. Sengstock (Imperial Colledge Press, London, 2015).

[76] Y. Ohashi, H. Tajima, and P. van Wyk, BCS-BEC crossover in cold atomic and in nuclear systems, Prog. Part. Nucl. Phys. 111, 103739 (2020).

[77] I. Danshita and L. Mathey, Counterflow superfluid of polaron pairs in Bose-Fermi mixtures in optical lattices, Phys. Rev. A 87, 021603(R) (2013).

[78] P. Siegl, S. I. Mistakidis, and P. Schmelcher, Many-body expansion dynamics of a Bose-Fermi mixture confined in an optical lattice, Phys. Rev. A 97, 053626 (2018).

[79] H. Mori, Transport, Collective motion, and Brownian motion, Prog. Theor. Phys. 33, 423 (1965).

[80] R. Zwanzig, Nonequilibrium Statistical Mechanics (Oxford University Press, New York, 2001). 